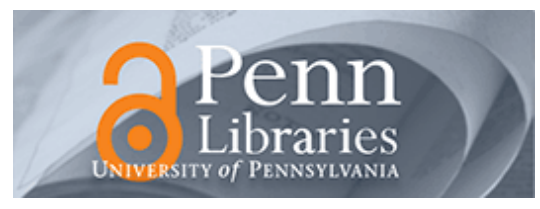

University of Pennsylvania

ScholarlyCommons

Business Economics and Public Policy Papers

Wharton Faculty Research

$1-2005$

\title{
Co-Evolution of Firms and Consumers and the Implications for Market Dominance
}

Joseph E. Harrington Jr

University of Pennsylvania

Myong-Hun Chang

Cleveland State University

Follow this and additional works at: https://repository.upenn.edu/bepp_papers

Part of the Business Commons, Economics Commons, and the Public Affairs, Public Policy and Public Administration Commons

\section{Recommended Citation}

Harrington, J. E., \& Chang, M. (2005). Co-Evolution of Firms and Consumers and the Implications for Market Dominance. Journal of Economic Dynamics and Control, 29 (1-2), 245-276. http://dx.doi.org/ 10.1016/j.jedc.2003.04.012

At the time of publication, author Joseph E. Harrington Jr. was affiliated with the John Hopkins University. Currently, he is a faculty member in the Business, Economics, Public Policy Department of the Wharton School at the University of Pennsylvania.

This paper is posted at ScholarlyCommons. https://repository.upenn.edu/bepp_papers/10

For more information, please contact repository@pobox.upenn.edu. 


\title{
Co-Evolution of Firms and Consumers and the Implications for Market Dominance
}

\author{
Abstract \\ Consider a setting in which firms randomly discover new ideas that affect their products or services and \\ implement favorable ones. At the same time that firms are adapting their offerings, consumers are \\ searching among firms for the best match. It is shown that implicit in these dual dynamics is an \\ increasing returns mechanism which can result in one firm dominating the market in the long run. The \\ conditions under which there is sustained market dominance are characterized.

\section{Keywords} \\ market dominance, innovation, search

\section{Disciplines} \\ Business | Economics | Public Affairs, Public Policy and Public Administration

\section{Comments} \\ At the time of publication, author Joseph E. Harrington Jr. was affiliated with the John Hopkins University. \\ Currently, he is a faculty member in the Business, Economics, Public Policy Department of the Wharton \\ School at the University of Pennsylvania.
}




\title{
Co-Evolution of Firms and Consumers and the Implications for Market Dominance*
}

\author{
Joseph E. Harrington, Jr. \\ Department of Economics \\ The Johns Hopkins University \\ Baltimore, MD 21218 \\ 410-516-7615, -7600 (Fax) \\ joe.harrington@jhu.edu \\ www.econ.jhu/people/harrington
}

\author{
Myong-Hun Chang \\ Department of Economics \\ Cleveland State University \\ Cleveland, OH 44115 \\ 216-687-4523, -9206 (Fax) \\ m.chang@csuohio.edu \\ www.csuohio.edu/changm
}

January 2001. Revision, September 2001.

\begin{abstract}
Consider a setting in which firms randomly discover new ideas that affect their products or services and implement only those ideas that increase current profit. At the same time that firms are adapting their offerings, consumers are searching among firms for the best match. It is shown that implicit in these dual dynamics is an increasing returns mechanism which can result in one firm dominating the market in the long run. The conditions under which there is sustained market dominance are characterized.
\end{abstract}

*The comments of Rob Axtell, Jimmy Chan, Leonard Cheng, Sigal Leviatan, and seminar participants at the University of Houston, Johns Hopkins University, University of Hong Kong, and Hong Kong University of Science and Technology and conference participants at the 2001 North American Summer Econometric Society Meetings are gratefully acknowledged. This research is supported by the National Science Foundation through Grant SES-0078752. 


\section{Introduction}

Consider the following scenarios.

- Stores in a geographic market compete by adopting new practices. Upon discovery, a store manager experiments with a new practice so as to assess its profitability. A new practice which raises profit is retained and otherwise is discarded. At the same time that stores are adapting their practices, consumers are searching among stores to find the one whose practices best conform with their preferences.

- Firms compete by modifying their products. Brand managers discover new product attributes which they adopt and sell in test markets. Those modifications that increase profit are retained and rolled out for the general market and the unprofitable ones are discarded. At the same time that firms are modifying their products, consumers are trying different products to find the best match.

- Internet sites compete by upgrading their site. Through online surveys and the tracking of clickstream behavior of those who visit their site, an online company learns about the preferences of visitors. Based on the information they've collected, a site evaluates new ideas and implements those that raise profit (or reduce losses). At the same time that sites are adapting their services, consumers are surfing among sites to find the one they like best.

What are the implications of these dual dynamics - firms adapting their offerings and consumers sorting themselves among firms - for market dominance? If one firm initially has a better store or product or Internet site and thereby attracts a bigger share of the market, does it have a higher likelihood of being dominant in the future? If market dominance is achieved, how easily is it sustained? How does the rate of consumer experimentation affect the persistence of market dominance?

In addressing these questions, this paper makes two contributions. First, it identifies a new source of increasing returns predicated on the property that a firm's best source of information about the value of a new idea is its current customer base. The right customer mix leads a firm to adopt the right kind of ideas which induces consumer sorting that generates an even better customer mix leading the firm to adopt even better ideas. While this feedback system is based upon a firm's customer mix, as opposed to market share as in most other increasing returns mechanisms, this will ultimately lead to dominance as measured by market share. The second contribution is exploring when this increasing returns mechanism generates sustained market dominance - one firm persistently having a higher market share. Analysis is performed on two models. In the first model, firms' offerings are differentiated horizontally and innovation takes the form of a new set of attributes in this space. We show that, regardless of the rate of consumer experimentation, sustained market dominance occurs with probability one. The model is then adapted to also allow the 
quality of firms' offerings to differ and be stochastic. If the maximum quality differential is sufficiently low, the first result persists and sustained market dominance is inevitable. If it is sufficiently high then sustained market dominance does not occur so that the identity of the market leader never gets locked in.

There is a well-developed literature identifying and exploring various feedback mechanisms that generate increasing returns. Learning-by-doing can create increasing returns because higher cumulative production results in lower marginal cost which may induce the firm to price lower and thereby have higher output. That higher output further increases its advantage in terms of cumulative production and thus lowers marginal cost even further. Cabral and Riordan (1994) characterize conditions whereby learning-by-doing does result in increasing market dominance. Another wellknown source of increasing returns is network externalities. Network externalities are present when the value of a product or service to a consumer is increasing in how many other consumers use it. A firm that initially has a high share of users will offer a more appealing product and thereby tend to have an even higher share of users in the future (see, for example, Katz and Shapiro, 1985, and Farrell and Saloner, 1986). A third source of increasing returns is explored by Bagwell, Ramey and Spulber (1997). Motivated by retail chains, they consider a setting in which a firm with higher sales has a greater incentive to invest in reducing marginal cost which leads it to set a lower price; thereby generating yet higher sales and a yet greater incentive to engage in cost-reducing investment. A closely related literature explores the extent to which a cost or demand leader invests more aggressively in innovation so as to maintain that leadership. Vickers (1986) explored the extent to which a firm with an existing cost advantage is inclined to spend more than other firms for the next innovation and thereby enhance its cost advantage. The current market leader maintained its advantage depending on the form of competition in the product market. In a more general setting, Budd, Harris, and Vickers (1993) characterized conditions under which a leader would tend to increase its advantage.

\section{Model}

There are two firms: firm 1 and firm 2. At any point in time, a firm has a location in $[0,1]$ which represents its practices. Let $x_{i}^{t}$ denote the practices or location of firm $i$ in period $t$. Time is discrete and unbounded so that $t=1,2, \ldots$ Initial locations are assumed to be different: $x_{1}^{0} \neq x_{2}^{0}{ }^{1}{ }^{1}$ There is a continuum of consumers who have preferences over practices and a consumer is defined by his ideal set of practices. For simplicity, there are only two types of consumers. A type 0 consumer's ideal location is 0 and a type 1 consumer's ideal location is 1 . A fraction $\alpha \in(.5,1)$ of consumers are type 0 . The type 0 consumer should be thought of as the typical consumer in this market and type 1 consumers as representing more of a niche sub-market.

\footnotetext{
${ }^{1}$ Results can be extended to when $x_{1}^{0}=x_{2}^{0}$ though it would involve additional tedious steps.
} 
At any point in time, a consumer is "loyal" to one of the two firms. This means that he buys with probability $1-\rho$ from the firm to which he is loyal and buys from the other firm with probability $\rho \in(0,1 / 2)$. One can think of $\rho$ as the rate of consumer search but also as being driven by exogenous forces disturbing a consumer's standard behavior; for example, a consumer might happen to be near his less favored store on a particular day and buys from it. ${ }^{2}$

The profit to a firm with practices $x$ generated by a type $k$ customer is specified to be $g(|k-x|)$ which is assumed to be a decreasing concave function of $|k-x|$.

A1 $g:[0,1] \rightarrow R_{+}$is twice continuously differentiable.

A2 $g^{\prime}(0)=0, g^{\prime}(d)<0 \forall d \in(0,1]$, and $g^{\prime \prime}(d)<0 \forall d \in[0,1]$.

Let $\pi(x, w(0), w(1))$ denote the profit to a firm when its practices are $x$ and it has a mass $w(0)$ of loyal type 0 customers and a mass $w(1)$ of loyal type 1 customers.

$$
\begin{aligned}
\pi(x, w(0), w(1))= & {[(1-\rho) w(0)+\rho(\alpha-w(0))] g(x) } \\
& +[(1-\rho) w(1)+\rho(1-\alpha-w(1))] g(1-x) .
\end{aligned}
$$

In explaining the profit function, a firm with $w(0)$ loyal type 0 customers finds a fraction $1-\rho$ of them buying from it in the current period and a fraction $\rho$ of the $\alpha-w(0)$ type 0 consumers who are loyal to the other firm. The total mass of type 0 consumers visiting this firm is then $[(1-\rho) w(0)+\rho(\alpha-w(0))]$ and from each of them the firm earns profit of $g(x)$.

In Lemma $1, \phi(w(0), w(1))$ is defined to be the location that maximizes current profit given a firm's loyal customer base is $(w(0), w(1))$. It shows that the optimal firm location is well-defined and is decreasing in the mass of type 0 loyal customers and increasing in the mass of type 1 loyal customers. Proofs are in the Appendix.

Lemma $1 \exists \phi:[0, \alpha] \times[0,1-\alpha] \rightarrow[0,1]$ such that

$$
\phi(w(0), w(1)) \in \arg \max \pi(x, w(0), w(1)) .
$$

$\phi$ is unique, $\frac{\partial \phi}{\partial w(0)}<0$, and $\frac{\partial \phi}{\partial w(1)}>0$.

Define $\phi \equiv \phi(\alpha, 0)$ and $\bar{\phi} \equiv \phi(0,1-\alpha)$ as the optimal location when a firm's loyal customers are all of the type 0 consumers and all of the type 1 consumers, respectively. By Lemma 1, it follows that $\phi(w(0), w(1)) \in[\phi, \bar{\phi}] \forall(w(0), w(1))$.

Notationally, it'll be easier to work with the following description of the state. Let $\beta^{t}$ and $\gamma^{t}$ denote the mass of type 0 consumers and type 1 consumers, respectively, that are loyal to firm 1 . Firm 1's profit in period $t$ is then

$$
\pi_{1}\left(x_{1}^{t}, \beta^{t}, \gamma^{t}\right)=\left[(1-\rho) \beta^{t}+\rho\left(\alpha-\beta^{t}\right)\right] g\left(x_{1}^{t}\right)+\left[(1-\rho) \gamma^{t}+\rho\left(1-\alpha-\gamma^{t}\right)\right] g\left(1-x_{1}^{t}\right),
$$

\footnotetext{
${ }^{2}$ Results are robust to allowing $\rho$ to vary over time, either deterministically or stochastically. What is important, however, is that $\rho$ is bounded above zero which seems quite reasonable.
} 
and for firm 2 is

$\pi_{2}\left(x_{2}^{t}, \beta^{t}, \gamma^{t}\right)=\left[(1-\rho)\left(\alpha-\beta^{t}\right)+\rho \beta^{t}\right] g\left(x_{2}^{t}\right)+\left[(1-\rho)\left(1-\alpha-\gamma^{t}\right)+\rho \gamma^{t}\right] g\left(1-x_{2}^{t}\right)$.

Define $\phi_{1}\left(\beta^{t}, \gamma^{t}\right) \equiv \phi\left(\beta^{t}, \gamma^{t}\right)$ and $\phi_{2}\left(\beta^{t}, \gamma^{t}\right) \equiv \phi\left(\alpha-\beta^{t}, 1-\alpha-\gamma^{t}\right)$.

Firm $i$ enters period $t$ with practices $x_{i}^{t-1}$. The discovery of alternative locations is presumed to be an act of creativity. Contrary to the usual assumption that the space of locations is known, we assume that it is unknown and innovation involves identifying points in that space. More specifically, in each period, a firm comes up with a new set of practices with probability $\omega \in(0,1) .{ }^{3}$ For period $t$, these new practices are denoted $y_{i}^{t}$ and are drawn from $[0,1]$ according to the cdf $F$.

A3 $F:[0,1] \rightarrow[0,1]$ is continuous and strictly increasing.

A firm decides either to discard the idea, in which case $x_{i}^{t}=x_{i}^{t-1}$, or to adopt it, in which case $x_{i}^{t}=y_{i}^{t}$. This decision is based upon whether its adoption would raise current profit; that is, myopic hill-climbing. Given a new idea of $y_{i}^{t}$, the dynamic on firm practices is then:

$$
x_{i}^{t}= \begin{cases}x_{i}^{t-1} & \text { if } \pi_{i}\left(x_{i}^{t-1}, \beta^{t}, \gamma^{t}\right) \geq \pi_{i}\left(y_{i}^{t}, \beta^{t}, \gamma^{t}\right) \\ y_{i}^{t} & \text { if } \pi_{i}\left(x_{i}^{t-1}, \beta^{t}, \gamma^{t}\right)<\pi_{i}\left(y_{i}^{t}, \beta^{t}, \gamma^{t}\right)\end{cases}
$$

The idea behind this rule is that a firm adopts an idea based on information about its appeal to those consumers who have recently bought from it. Returning to our motivating examples in the Introduction, the interpretation is as follows. One could imagine a store or brand manager implementing a new idea for a period of experimentation which serves to reveal its profitability. What is implicitly assumed is that the length of the period of experimentation is small so that it can be ignored. ${ }^{4}$ For the case of an Internet site, one can presume it has accumulated information about who has recently visited its site and this information is used to judge the value of an idea. At the heart of all of these examples is that those consumers who buy or visit a firm are the best (and perhaps only) source of information about what are worthwhile ideas. This provides the critical link between a firm's customer base and the set of new ideas that it would adopt. ${ }^{5}$

It is straightforward to characterize the set of acceptable ideas. Given a current location $x$ and loyal customers $(w(0), w(1))$, it follows from the strict concavity of $\pi$ that there is a connected set of locations which yield at least as high a level of profit as is achieved with $x$. One extreme point of this set is $x$. The other extreme

\footnotetext{
${ }^{3}$ Assuming $\omega<1$ simplies some steps in the proofs. All results go through if $\omega=1$.

${ }^{4}$ This type of dynamic is also used in Gale and Rosenthal (1999) where they provide the same interpretation for what they refer to as virtual experimentation.

${ }^{5}$ Though firms are not permitted to recall previously discovered ideas, if memory is bounded, so a firm could only retain some maximal number of ideas, we do not think that it would affect our main results.
} 
point, denoted $\psi(x, w(0), w(1))$, is defined by $\pi(\psi(x, w(0), w(1)), w(0), w(1))=$ $\pi(x, w(0), w(1))$ (see Figure 1). ${ }^{6}$ Define $\psi_{1}(x, \beta, \gamma) \equiv \psi(x, \beta, \gamma)$ and $\psi_{2}(x, \beta, \gamma) \equiv$ $\psi(x, \alpha-\beta, 1-\alpha-\gamma)$. The set of acceptable ideas in period $t$, and also the set of possible values for $x_{i}^{t}$, is then

$$
\left[\min \left\{\psi_{i}\left(x_{i}^{t-1}, \beta^{t}, \gamma^{t}\right), x_{i}^{t-1}\right\}, \max \left\{\psi_{i}\left(x_{i}^{t-1}, \beta^{t}, \gamma^{t}\right), x_{i}^{t-1}\right\}\right] .
$$

Next consider the equation of motion on a firm's loyal customers. A consumer who is loyal to firm $i$ in period $t$ and buys from firm $i$ in period $t$ is assumed to maintain his loyalty into period $t+1$. A type $k$ consumer who is loyal to firm $i$ in period $t$ and buys from store $j$ in period $t$ maintains his loyalty into period $t+1$ iff $\left|x_{i}^{t-1}-k\right| \leq\left|x_{j}^{t}-k\right|$ and is loyal to firm $j$ otherwise. The idea is that a consumer's loyalty is based on how close a firm's practices are to his ideal practices. If a consumer loyal to firm $i$ bought from it in period $t-1$ but experimented with the other firm in period $t$ then the consumer is assumed to make this judgement by comparing his most recent experiences. To ensure that this is the preceding period for a consumer's favored firm, it is assumed that if a consumer experimented in period $t$ but did not change his loyalty then he does not experiment in period $t$. A consumer's information is then no more than one period old. The resulting equations of motion on loyal customers are: ${ }^{7}$

$$
\begin{array}{r}
\beta^{t+1}= \begin{cases}\beta^{t}+\rho\left(\alpha-\beta^{t}\right) & \text { if } x_{1}^{t-1}<x_{2}^{t} \text { and } x_{1}^{t}<x_{2}^{t-1} \\
\beta^{t} & \text { if } x_{1}^{t-1}<x_{2}^{t} \text { and } x_{2}^{t-1}<x_{1}^{t} \\
(1-\rho) \beta^{t}+\rho\left(\alpha-\beta^{t}\right) & \text { if } x_{2}^{t}<x_{1}^{t-1} \text { and } x_{1}^{t}<x_{2}^{t-1} \\
(1-\rho) \beta^{t} & \text { if } x_{2}^{t}<x_{1}^{t-1} \text { and } x_{2}^{t-1}<x_{1}^{t}\end{cases} \\
\gamma^{t+1}= \begin{cases}\gamma^{t}+\rho\left(1-\alpha-\gamma^{t}\right) & \text { if } x_{2}^{t}<x_{1}^{t-1} \text { and } x_{2}^{t-1}<x_{1}^{t} \\
\gamma^{t} & \text { if } x_{2}^{t}<x_{1}^{t-1} \text { and } x_{1}^{t}<x_{2}^{t-1} \\
(1-\rho) \gamma^{t}+\rho\left(1-\alpha-\gamma^{t}\right) & \text { if } x_{1}^{t-1}<x_{2}^{t} \text { and } x_{2}^{t-1}<x_{1}^{t} \\
(1-\rho) \gamma^{t} & \text { if } x_{1}^{t-1}<x_{2}^{t} \text { and } x_{1}^{t}<x_{2}^{t-1}\end{cases}
\end{array}
$$

For example, if $x_{2}^{t}<x_{1}^{t-1}$ then type 0 customers who are loyal to firm 1 and buy from firm 2 switch loyalty to firm 2 . Thus, firm 1 only retains $1-\rho$ of its $\beta^{t}$ type 0 consumers who were loyal to it in the previous period. If, in addition, $x_{1}^{t}<x_{2}^{t-1}$ then type 0 customers who are loyal to firm 2 and buy from firm 1 will switch loyalty to firm 1 . There are $\rho\left(\alpha-\beta^{t}\right)$ such consumers. As a result, when $x_{2}^{t}<x_{1}^{t-1}$ and $x_{1}^{t}<x_{2}^{t-1}$, firm 1's set of type 0 loyal customers is $(1-\rho) \beta^{t}+\rho\left(\alpha-\beta^{t}\right)$. Finally, assume interior values for the initial mix of consumers: $\beta^{1} \in(0, \alpha), \gamma^{1} \in(0,1-\alpha) .^{8}$

\footnotetext{
${ }^{6}$ When $\nexists x^{\prime} \in[0,1]$ such that $\pi\left(x^{\prime}, w(0), w(1)\right)=\pi(x, w(0), w(1))$ then $\psi=0$ if $\phi<x$ and $\psi=1$ if $\phi>x$.

${ }^{7}$ Note that the equations of motion are only defined for when locations are different. These could easily be extended to include identical locations though it'll prove irrelevant since if $x_{1}^{0} \neq x_{2}^{0}$ then $x_{1}^{t} \neq x_{2}^{t} \forall t$ with probability one.

${ }^{8}$ Results are robust to this assumption though amending the proofs would involve handling additional cases.
} 
Let us offer a few comments on these behavioral assumptions. Assuming firms adopt new practices on the basis of current profit is most appropriate for environments in which firms have little information about how consumers behave and the distribution of consumer types. In that case, firm behavior is likely to be experientially-based - they adopt practices that experience (or experimentation) tells them are good ones. ${ }^{9}$ This is to be contrasted with an equilibrium approach in which firms have an accurate model of the market based on their beliefs over the distribution of consumer types and how consumers respond to practices. The assumptions that consumers base their loyalty on their most recent experiences and experiment at an exogenous rate seem quite reasonable. Having behavior be forward-looking would require consumers to formulate a model of how firms adjust their practices over time as well as how other consumers are determining their loyalty. As the development of such a model is likely to be quite costly, it is arguably reasonable for consumers to use the type of heuristics specified here. ${ }^{10}$

These dual dynamics create a feedback system defined on the state variables $\left(x_{1}^{t-1}, x_{2}^{t-1}, \beta^{t}, \gamma^{t}\right)$. The dynamic on firm practices in (5) depends on the current allocation of customer loyalty across firms. The allocation of customer loyalty, as specified in (6)-(7), depends on stores' practices. To derive some initial insight into why increasing returns may emerge from this feedback system, let $H_{i}\left(\cdot \mid x_{i}^{t-1}, \beta^{t}, \gamma^{t}\right)$ denote the cdf on $x_{i}^{t} . H_{i}\left(\cdot \mid x_{i}^{t-1}, \beta^{t}, \gamma^{t}\right)$ depends on the probability of a firm receiving a new idea, $\omega$, the probability distribution over new ideas, $F$, and the rule for adjusting store practices, (5). In Lemma $2, K>_{1} L$ means that cdf $K$ stochastically dominates cdf $L$ in the first-degree.

Lemma 2 If $\widetilde{\beta} \geq \bar{\beta}$ and $\widetilde{\gamma} \leq \bar{\gamma}$ then $H_{1}\left(\cdot \mid x_{1}^{t-1}, \bar{\beta}, \bar{\gamma}\right)>_{1} H_{1}\left(\cdot \mid x_{1}^{t-1}, \widetilde{\beta}, \widetilde{\gamma}\right)$ and $H_{2}\left(\cdot \mid x_{2}^{t-1}, \widetilde{\beta}, \widetilde{\gamma}\right)>_{1} H_{2}\left(\cdot \mid x_{2}^{t-1}, \bar{\beta}, \bar{\gamma}\right)$.

Lemma 2 says that if firm 1's period $t$ share of type 0 consumers is increased and/or its share of type 1 consumers is decreased, more probability is assigned to lower values for $x_{1}^{t}$ and to higher values for $x_{2}^{t}$. Hence, the probability that $x_{1}^{t}<x_{2}^{t-1}$, so that type 0 consumers switch loyalty from firm 2 to firm 1 , increases and the probability that $x_{1}^{t-1}<x_{2}^{t}$, so that type 0 consumers who are loyal to firm 1 maintain

\footnotetext{
${ }^{9}$ The behavioral assumption is that firms, as well as consumers, are procedurally rational in the sense that they do not anticipate the behavior of other agents but rather simply associate particular consequences with particular actions and choose the action yielding the best consequence. Developed by Simon (1982), procedural rationality has recently been explored in Rubinstein (1998).

${ }^{10}$ It is worth noting that the random element in the model are firms' practices rather than consumers' loyalty decisions. This choice is motivated by our sense that while there may be some randomness in an individual consumer's loyalty decision that the law of large numbers would tend to operate at the level of the firm populations. That is, it is unlikely that a firm would experience a substantive change in its customer base due to random actions by consumers. In contrast, we believe that innovation is highly stochastic; there is a fair amount of randomness associated with coming up with new ideas.
} 
their loyalty, increases. As a lower location makes a firm more attractive to type 0 consumers and less attractive to type 1 consumers, $\beta^{t+1}$ will tend to be higher and $\gamma^{t+1}$ lower when $\left(\beta^{t}, \gamma^{t}\right)=(\widetilde{\beta}, \widetilde{\gamma})$ than when $\left(\beta^{t}, \gamma^{t}\right)=(\bar{\beta}, \bar{\gamma})$. In this way, a customer mix biased toward type 0 consumers induces a firm to adopt ideas more suitable for type 0 consumers which tends to cause the customer mix to become more biased to type 0 consumers and thereby further induces the adoption of such ideas. Lemma 2 also implies that the probability that $x_{1}^{t}<x_{2}^{t}$, so that firm 1's new location is more desirable to type 0 consumers than firm 2's new location, is higher under $(\widetilde{\beta}, \widetilde{\gamma})$ than $(\bar{\beta}, \bar{\gamma}) \cdot{ }^{11}$ To what extent does this feedback create increasing returns and generate sustained market dominance? Given that firms are always receiving new ideas, can a sufficiently high rate of consumer experimentation ensure that the current market leader will not become entrenched? These are some of the questions that will be addressed in the remainder of the paper.

While this particular feedback system between firms and consumers is new, previous work has modelled the dynamical movement of buyers among sellers. In Bergemann and Välimäki (1997), a new firm's product is of unknown quality and both buyers and sellers receives signals, the informativeness of which is increasing in the number of units sold of the product. Though a very different setting, there is a similarity as buyers move among sellers as they learn about the new seller's quality. In the search model of Burdett and Coles (1997), consumers know the price distribution in the market but not the price that each firm charges. Consumers enter the market and engage in costly price search (products are homogeneous). In each period, a firm has a stock of regular customers who are defined to be those that bought from it last period. A regular customer avoids incurring a search cost while to buy from any other firm requires incurring a search cost. This gives a firm some market power over its regular customers and causes it to price higher when its stock of regular customers is larger relative to the flow of non-regular searching customers who visit it. A higher price generates more profit from its regular customers but results in the firm being less successful in inducing searching consumers to become regular ones. There is then a feedback mechanism present as a firm's stock of regular customers influences its price which determines next period's stock of regular customers. Note the similarity with our model where a firm's stock of loyal customers influences the practices that a firm wants to adopt and those practices determine next period's stock of loyal customers. Finally, Weisbuch, Kirman, and Herreiner (2000) explore the extent to which buyer and sellers form long-lasting relationships. In each period, buyers decide which

${ }^{11}$ The probability that $x_{1}^{t}<x_{2}^{t}$ is $\int_{0}^{1} H_{1}(x) d H_{2}(x)$. The statement is then true iff

$$
\int_{0}^{1} \widetilde{H}_{1}(x) d \widetilde{H}_{2}(x) \geq \int_{0}^{1} \bar{H}_{1}(x) d \bar{H}_{2}(x) .
$$

This inequality holds because $\widetilde{H}_{2}$ stochastically dominates $\bar{H}_{2}$ in the first degree and $\widetilde{H}_{1}(x) \geq$ $\bar{H}_{1}(x) \forall x$. 
seller to visit using reinforcement learning; the probability of visiting a seller depends on the past profit realized by interacting with that seller. Profit from a transaction depends on whether the seller has the supply to sell to a buyer. Depending on the variant of the model, sellers are either fixed in their behavior or choose to supply an amount equal to the previous period's demand.

\section{Nash Equilibrium}

Prior to analyzing the dynamic model of the preceding section, it is useful to characterize Nash equilibrium for the complete information game as a benchmark. Imagine that firms are aware of the distribution of consumers, the rate at which consumers buy from them, and know how to implement each practice in $[0,1]$. Thus, contrary to the preceding model, it is assumed that all that could be known about how to satisfy consumers is known. A firm is modelled as choosing a practice to maximize its profit given the (correctly) anticipated practice of the other firm and the (correctly) anticipated sorting by consumers. Firm $i$ 's payoff is then ${ }^{12}$

$$
\pi_{i}\left(x_{1}, x_{2}\right)= \begin{cases}(1-\rho) \alpha g\left(x_{i}\right)+\rho(1-\alpha) g\left(1-x_{i}\right) & \text { if } x_{i}<x_{j} \\ (\alpha / 2) g\left(x_{i}\right)+[(1-\alpha) / 2] g\left(1-x_{i}\right) & \text { if } x_{i}=x_{j} \\ \rho \alpha g\left(x_{i}\right)+(1-\rho)(1-\alpha) g\left(1-x_{i}\right) & \text { if } x_{j}<x_{i}\end{cases}
$$

By locating to the left (right) of its competitor, a firm induces all type 0 (1) consumers to be loyal to it. If it locates exactly at the other firm's location then the two firms equally divide the set of consumers.

Theorem 3 shows that if the proportion of type 0 consumers is sufficiently high then an equilibrium exists. Furthermore, the equilibrium is unique and has both firms deploying the ideal practice for type 0 consumers and thereby sharing the market.

Theorem $3 \exists \underline{\alpha} \in(1 / 2,1)$ such that: i) if $\alpha \in(1 / 2, \underline{\alpha})$ then a pure-strategy Nash equilibrium does not exist; and ii) if $\alpha \in[\underline{\alpha}, 1]$ then $\left(x_{1}, x_{2}\right)=(0,0)$ is the unique pure-strategy Nash equilibrium.

First note that if firms have different locations, say $x_{1}<x_{2}$, so firm 1 is attracting type 0 consumers and firm 2 is attracting type 1 consumers, firm 2 can improve its profit by locating just to the left of $x_{1}$ and attracting type 0 consumers because there are more of them than type 1 consumers. The only way that cannot happen is if $x_{1}=0$. However, if $\alpha$ is sufficiently close to $1 / 2$ then firm 2 prefers to locate at $\bar{\phi}$ (the optimal location when all of its loyal customers are type 1) and focus on serving type 1 consumers then to locate at 0 and share both consumer types. But if it does that then firm 1 prefers to locate at $\underline{\phi}$ (the optimal location when all of its loyal

\footnotetext{
${ }^{12}$ To make an appropriate comparison with the dynamical model, $\rho$ is maintained and not set equal to zero.
} 
customers are type 0). Hence, an equilibrium does not exist when $\alpha$ is low. When $\alpha$ is sufficiently high then both firms are content to locate at 0 and share the market rather than be the exclusive preferred provider for the minority consumer type.

\section{Sustained Market Dominance}

We will use the term "dominance" to refer to one firm having more than half of the market or, more specifically, having almost all type 0 consumers as loyal customers. This section has three results. Theorem 4 derives the set of states for which a particular firm dominates for sure. Theorem 5 derives the set of states for which either firm has a positive probability of dominating. Finally, Theorem 6 establishes that the model always generates sustained market dominance.

The first result characterizes a set of states such that a particular firm dominates in the long-run for sure. As defined below, $\Omega_{i}$ is the set of states such that firms' sets of acceptable ideas do not intersect and firm $i$ 's maximal acceptable idea is less than firm $j$ 's minimal acceptable idea. See Figure 2 for an example of a state in $\Omega_{1}$.

$$
\begin{gathered}
\Omega_{1} \equiv\left\{\left(x_{1}, x_{2}, \beta, \gamma\right): \max \left\{\psi_{1}\left(x_{1}, \beta, \gamma\right), x_{1}\right\}<\min \left\{\psi_{2}\left(x_{2}, \beta, \gamma\right), x_{2}\right\}\right\} \\
\Omega_{2} \equiv\left\{\left(x_{1}, x_{2}, \beta, \gamma\right): \max \left\{\psi_{2}\left(x_{2}, \beta, \gamma\right), x_{2}\right\}<\min \left\{\psi_{1}\left(x_{1}, \beta, \gamma\right), x_{1}\right\}\right\} .
\end{gathered}
$$

Theorem 4 shows that if the state is in $\Omega_{i}$ then firm $i$ dominates for sure. Once in $\Omega_{1}$ or $\Omega_{2}$, the dynamic path on market shares is deterministic with the dominant firm steadily attracting more type 0 consumers and steadily losing type 1 consumers. However, as shown in the proof, the path on firms' practices remains stochastic and, furthermore, each firm's practice will generally not be monotonic. Probabilistically, practices converge to either $\left(x_{1}, x_{2}\right)=(\underline{\phi}, \bar{\phi})$, if the current state is in $\Omega_{1}$, or $\left(x_{1}, x_{2}\right)=$ $(\bar{\phi}, \underline{\phi})$, if the current state is in $\Omega_{2}$.

Theorem 4 If $\left(x_{1}^{t-1}, x_{2}^{t-1}, \beta^{t}, \gamma^{t}\right) \in \Omega_{1}$ then with probability one:

$$
\begin{array}{lll}
\beta^{\tau}=(1-\rho)^{\tau-t} \beta^{t}+\left[1-(1-\rho)^{\tau-t}\right] \alpha & \\
\gamma^{\tau}=(1-\rho)^{\tau-t} \gamma^{t} & \forall \tau \geq t
\end{array}
$$

and $\lim _{\tau \rightarrow \infty}\left(\beta^{\tau}, \gamma^{\tau}\right)=(\alpha, 0)$. If $\left(x_{1}^{t-1}, x_{2}^{t-1}, \beta^{t}, \gamma^{t}\right) \in \Omega_{2}$ then with probability one:

$$
\begin{aligned}
& \beta^{\tau}=(1-\rho)^{\tau-t} \beta^{t} \\
& \gamma^{\tau}=(1-\rho)^{\tau-t} \gamma^{t}+\left[1-(1-\rho)^{\tau-t}\right](1-\alpha) \quad \forall \tau \geq t
\end{aligned}
$$

and $\lim _{\tau \rightarrow \infty}\left(\beta^{\tau}, \gamma^{\tau}\right)=(0,1-\alpha)$.

If the state is in $\Omega_{1}$ then it implies that $x_{1}^{t-1}<x_{2}^{t-1}$ so that type 0 consumers prefer firm 1's practices. If this ordering of firms' locations persists then, due to continual 
consumer experimentation, all consumers will eventually learn that firm 1 better meets the needs of type 0 consumers while firm 2 better serves type 1 consumers. Eventually all type 0 consumers will be loyal to firm 1 and all type 1 consumers will be loyal to firm 2. The next issue is what ensures that the ordering of firms' locations persists. Note that firm 1 does not adopt any idea in period $t$ which exceeds $\max \left\{\psi_{1}\left(x_{1}^{t-1}, \beta^{t}, \gamma^{t}\right), x_{1}^{t-1}\right\}$ and firm 2 does not adopt any idea which is less than $\min \left\{\psi\left(x_{2}^{t-1}, \beta^{\tau}, \gamma^{\tau}\right), x_{2}^{t-1}\right\}$. Since

$$
\max \left\{\psi_{1}\left(x_{1}^{t-1}, \beta^{t}, \gamma^{t}\right), x_{1}^{t-1}\right\}<\min \left\{\psi\left(x_{2}^{t-1}, \beta^{\tau}, \gamma^{\tau}\right), x_{2}^{t-1}\right\}
$$

then $x_{1}^{t}<x_{2}^{t}$ so that this ordering is sure to continue into the next period. This is not sufficient to ensure the result, however, because $\psi_{1}\left(x_{1}^{t-1}, \beta^{t}, \gamma^{t}\right)$ is not monotonically decreasing over time and $\psi_{2}\left(x_{1}^{t-1}, \beta^{t}, \gamma^{t}\right)$ is not monotonically increasing over time and, therefore, firms' locations are not monotonic over time even when the state lies in $\Omega_{1}$. However, the proof of Theorem 4 shows that $\max \left\{\psi_{1}\left(x_{1}^{t-1}, \beta^{t}, \gamma^{t}\right), x_{1}^{t-1}\right\}$ is monotonically decreasing over time and $\min \left\{\psi\left(x_{2}^{t-1}, \beta^{\tau}, \gamma^{\tau}\right), x_{2}^{t-1}\right\}$ is monotonically increasing over time. This implies $x_{1}^{\tau}<x_{2}^{\tau} \forall \tau \geq t$ and therefore firm 1 will eventually have all type 0 consumers loyal to it.

Whether the market ends up being persistently dominated by one firm then depends on whether the state ever enters into $\Omega_{1} \cup \Omega_{2}$. The next result shows that if the state is not in $\Omega_{1} \cup \Omega_{2}$ then with positive probability it ends up in $\Omega_{1}$, so that firm 1 dominates, and with positive probability it ends up in $\Omega_{2}$, so that firm 2 dominates.

Theorem 5 If $\left(x_{1}^{t-1}, x_{2}^{t-1}, \beta^{t}, \gamma^{t}\right) \notin \Omega_{1} \cup \Omega_{2}$ then: i) $\lim _{\tau \rightarrow \infty}\left(\beta^{t+\tau}, \gamma^{t+\tau}\right)=(\alpha, 0)$ with positive probability; and ii) $\lim _{\tau \rightarrow \infty}\left(\beta^{t+\tau}, \gamma^{t+\tau}\right)=(0,1-\alpha)$ with positive probability.

Though sustained market dominance is a possible outcome, is it inevitable? Theorem 6 shows that, with probability one, one firm will ultimately achieve a dominant position that will never be relinquished.

Theorem 6 Almost surely, $\lim _{t \rightarrow \infty}\left(\beta^{t}, \gamma^{t}\right) \in\{(\alpha, 0),(0,1-\alpha)\}$.

In summarizing these results, first note that $\Omega_{1}$ and $\Omega_{2}$ are absorbing sets of states. If the state is in $\Omega_{i}$ then it stays there and, more specifically, the state converges to firm $i$ having all type 0 consumers as loyal customers and firm $j$ having all type 1 consumers as loyal customers. The long-run market share of firm $i$ is then $(1-\rho) \alpha+\rho(1-\alpha)$. If the state is outside of $\Omega_{1} \cup \Omega_{2}$ then eventually it will be in $\Omega_{1} \cup \Omega_{2}$ and, furthermore, it can be in either $\Omega_{1}$ or $\Omega_{2}$ with positive probability. Thus, if the state is not in $\Omega_{1} \cup \Omega_{2}$ then either firm could end up being dominant. ${ }^{13}$

\footnotetext{
${ }^{13}$ Define $\widehat{\phi}$ as the optimal location when a firm has half of all type 0 consumers and half of all type 1 consumers as loyal customers: $\widehat{\phi} \equiv \phi_{1}\left(\frac{\alpha}{2}, \frac{1-\alpha}{2}\right)$. If $\left(x_{1}^{t-1}, x_{2}^{t-1}, \beta^{t}, \gamma^{t}\right)=\left(\widehat{\phi}, \widehat{\phi}, \frac{\alpha}{2}, \frac{1-\alpha}{2}\right)$ then
} 
Implicit in our model is an increasing returns mechanism. A firm that currently has a customer mix biased toward the prevalent consumer type in the market will tend to identify as valuable those ideas well-suited to that type. Their adoption impacts future loyalty switching by consumers and, in particular, generally leads to a customer mix even more biased toward the prevalent type which leads a firm to adopt ideas more suitable for them. Eventually, this process results in one of the firms capturing and retaining most of the market. While the model is highly simplified, the underlying story seems quite general. It is predicated upon the idea that firms are uncertain about the value of a new idea and that their best source of information about its value are those consumers who are currently buying from it. Though there are indeed other sources of information - for example, market surveys - a firm's current customers will still be a primary source of information and, as long as that is the case, the increasing returns mechanism that has been identified would seem pertinent to understanding market dominance.

Note that myopic dynamics do not converge to a Nash equilibrium. Since $\lim _{t \rightarrow \infty}\left(\beta^{t}, \gamma^{t}\right) \in$ $\{(\alpha, 0),(0,1-\alpha)\}$ then, eventually, one firm's optimal location is converging to $\phi$ and the other's to $\bar{\phi}$. In a probabilistic sense, one firm's location is then converging to $\underline{\phi}$ and the other's to $\bar{\phi}$. Since $0<\phi<\bar{\phi}<1$, firms' long-run locations are interior. In contrast, Nash equilibrium, when it exists, has both firms locate at 0 . In that case, no single firm dominates as the two firms equally share the market. ${ }^{14}$ Further thought is required to determine why convergence to Nash equilibrium does not occur.

There is a vast literature exploring dynamics in games based on myopic or imitative behavior. In briefly reviewing this work, it is important to remember that myopia can come in many flavors. Fudenberg and Levine (1998) review a wide class of myopic dynamics for which non-Nash outcomes are typically unstable. ${ }^{15}$ The work of Kandori, Mailath, and Rob (1993) and Young (1993) found convergence to Nash equilibrium so the focus of their studies was equilibrium selection. The general tendency of myopic dynamics is to converge to a Nash equilibrium. Reviewing some work based on imitative dynamics within oligopoly theory reveals a variety of results. For

it is easy to show that, with probability one,

$$
\left(x_{1}^{\tau}, x_{2}^{\tau}, \beta^{\tau+1}, \gamma^{\tau+1}\right)=\left(\widehat{\phi}, \widehat{\phi}, \frac{\alpha}{2}, \frac{1-\alpha}{2}\right) \forall \tau \geq t .
$$

Hence, $\left(\widehat{\phi}, \widehat{\phi}, \frac{\alpha}{2}, \frac{1-\alpha}{2}\right)$ is a stable point for which there is no dominant firm. This case is excluded from Theorem 6 since we've assumed $x_{1}^{0} \neq x_{2}^{0}$. What Theorem 6 implies is that $\left(\widehat{\phi}, \widehat{\phi}, \frac{\alpha}{2}, \frac{1-\alpha}{2}\right)$ is not locally stable.

${ }^{14}(\underline{\phi}, \bar{\phi})$ is a "local" Nash equilibrium in the sense that each firm's strategy is a best response in a neighborhood of its strategy.

${ }^{15}$ For example, if fictitious play converges then it converges to a Nash equilibrium (Proposition 2.2) and any non-Nash outcome is unstable with respect to any behavioral rules that are asymptotically myopic and are based on beliefs that are asymptotically the same as the empirical distribution (Proposition 4.1). 
the quantity game with homogeneous goods, Vega-Redondo (1997) shows that the process converges to a price more competitive than equilibrium when a firm imitates the quantity of the most profitable firms. Similar results are derived in Rhode and Stegeman (2001) for the differentiated products price game and quantity game. For the Bertrand price game in which sellers imitate the price of the most profitable sellers, Hehenkamp, Qin, and Stuart (1999) and Alós-Ferrer, Ania, and Schenk-Hoppé (2000) find convergence to the Nash equilibrium. Hehenkamp (2000) modifies this model so that consumers only gradually learn about sellers' prices and shows that if consumers learn sufficiently slowly that the system does not converge to a Nash equilibrium.

\section{Comparative Dynamics and Simulations}

To explore the presence of a first-mover advantage and the form it might take, simulations were conducted. The term "first-mover advantage" is used to refer to any advantage emanating from the initial conditions to the system. The system has three parameter values: $\alpha$ (the proportion of type 0 consumers in the population), $\rho$ (the rate at which consumers experiment), and $\omega$ (the rate at which firms discovers new ideas); and four initial conditions: $\beta^{1}$ (the initial proportion of type 0 consumers loyal to firm 1), $\gamma^{1}$ (the initial proportion of type 1 consumers loyal to firm 1), $x_{1}^{0}$ (the initial location of firm 1), and $x_{2}^{0}$ (the initial location of firm 2). The function $g$ is specified to be: $g(|k-x|)=1-(k-x)^{2}$. In that case, the long-run locations are:

$$
\underline{\phi}=\frac{\rho(1-\alpha)}{(1-\rho) \alpha+\rho(1-\alpha)}, \bar{\phi}=\frac{(1-\rho)(1-\alpha)}{(1-\rho)(1-\alpha)+\rho(1-\alpha)} .
$$

Simulations involve a four step procedure. First, values are set for $\alpha, \rho, \omega, \beta^{1}$, and $\gamma^{1}$. Second, values for $x_{1}^{0}$ and $x_{2}^{0}$ are randomly selected from $[0,1]$ according to a uniform distribution. Third, the model is played out. This involves generating a sequence of ideas; in each period, with probability $\omega$ an idea is randomly selected from $[0,1]$ according to a uniform distribution. Given this sequence of ideas, firms and consumers act according to the equations of motion. Steps 2-3 are then repeated 1000 times. The values reported below are the averages of these 1000 runs. In some of the runs, step 2 is replaced with initial locations being fixed over the 1000 runs. Where that occurs, it is noted.

The first issue relates to identifying a first-mover advantage in this model. There are two obvious possibilities - the initial mix of type 0 consumers relative to type 1 consumers and the initial firm locations. The height of the surface in Figure 3 measures the frequency with which firm 1 dominates - that is, the state eventually enters into $\Omega_{1}$ so that the long-run market share of firm 1 is $(1-\rho) \alpha+\rho(1-\alpha)$. Its dependence on firm 1's initial share of type 0 consumers, $\beta^{1} / \alpha$, and its initial share of type 1 consumers, $\gamma^{1} /(1-\alpha)$, is shown. These results are for when $60 \%$ 
of consumers are type $0(\alpha=.6)$, a firm, on average, receives seven ideas every ten periods $(\omega=.7)$, and a consumer, on average, experiments once every ten periods $(\rho=.1)$ and once every five periods $(\rho=.2)$. Consistent with the intuition coming out of the equations of motion, a higher mass of loyal type 0 consumers and a lower mass of loyal type 1 consumers increases the frequency with which firm 1 dominates. By having an initial customer mix biased towards type 0 consumers, firm 1 is more inclined to adopt ideas suitable for type 0 consumers and this ultimately enhances the likelihood of dominating the market. Also note that firm 1's initial share of type 0 consumers could be quite low but it still is quite likely to dominate as long as the customer mix is biased towards type 0 consumers. For example, it dominates well more than $50 \%$ of the time when its initial share of type 0 consumers is only $20 \%$ but its share of type 1 consumers is only $10 \%$. Hence, it is not simply that more type 0 consumers is conducive to dominating but rather a higher proportion of type 0 consumers among one's loyal customer base leads to dominance.

While a bigger relative presence of type 0 consumers is conducive to being dominant, it is not true that an initial location closer to that most desired by type 0 consumers is always conducive to being dominant. Figure 4 reports the frequency with which firm 1 dominates as a function of the initial locations, $\left(x_{1}^{0}, x_{2}^{0}\right)=(x, 1-x)$, and firm 1's initial share of type 0 consumers. Parameter values and other initial conditions are set at: $\gamma^{1}=.5(1-\alpha), \alpha=.6, \omega=.7$, and $\rho \in\{.3, .4\}$. Starting from $x=.5$, so that firms are both initially located at .5, reducing $x$ so firm 1 is closer to 0 and firm 2 is farther away from 0 does enhance firm 1's prospects for dominance. Intuitively, a location closer to 0 is more effective at attracting type 0 consumers. However, when $x$ is too close to 0 , further decreases actually reduce the frequency with which firm 1 is dominant. To see why this occurs, hold fixed firm 1 's optimal location, $\phi_{1}^{1}$. If $x_{1}^{0}<\phi_{1}^{1}$ then the set of acceptable ideas is $\left[x_{1}^{0}, \psi_{1}^{1}\right]$. A decrease in $x_{1}^{0}$ will then serve to increase $\psi_{1}^{1}$, making it more likely that firm 1 would adopt higher locations and thus more likely that firm 2's location is more desirable to type 0 consumers. A very low location may be undesirable because if it is well below one's optimal location, indicating that a firm's current location is not in line with their current customer base, it may lead the firm to radically increase its location.

Another way in which to approach the issue of dominance is to characterize $\Omega_{i}$ which is the part of the state space for which firm $i$ dominates for sure. Once the state enters into $\Omega_{i}$ then firm $i$ 's market share steadily converges to $(1-\rho) \alpha+\rho(1-\alpha)$. Animation is provided at www. csuohio.edu/changm/main/research/dominance.htm which shows the projection of $\Omega_{1}$ (and $\left.\Omega_{2}\right)$ on the space of values for $\left(x_{1}^{t-1}, x_{2}^{t-1}, \beta^{t}\right)$ and depicts how this projection changes with respect to the fourth state variable, $\gamma^{t}$. The red points comprise $\Omega_{1}$ and the blue points comprise $\Omega_{2}$ (for a finite grid). Observing $\Omega_{1}$, the set of values for $\left(x_{1}^{t-1}, x_{2}^{t-1}\right)$ is larger for higher values for $\beta^{t}$ and this projection shrinks as $\gamma^{t}$ rises. Also note that as $\gamma^{t}$ rises, the projection of $\Omega_{1}$ gets concentrated on higher values for $\beta^{t}$ but moderates values for $x_{1}^{t-1}$ and $x_{2}^{t-1}$ consistent 
with the point made with Figure $4 .^{16}$

A second question to explore with simulations is to what extent the rate of consumer experimentation is complementary to a first-mover advantage. There are two countervailing forces at play. If, say, firm 1 has a higher mix of type 0 consumers then it is more likely than the other firm to adopt a location that is more attractive to those consumers. If consumers experiment at a higher rate, type 0 consumers who are currently loyal to the other store will then learn about the firm's superior practices and flow to it quicker. This makes it more likely that the state will get into $\Omega_{1}$. By this argument, a higher value for $\rho$ augments the first-mover advantage from a higher mix of type 0 consumers. On the other hand, if the current market laggard, in terms of the customer mix, is able to develop a superior practice then more consumer experimentation will result in a heavier flow of type 0 consumers to it. It may then be able to become a market leader before the other firm develops a yet even better practice. Examination of Figure 3 suggests that the latter effect dominates. As $\rho$ is increased from .1 to .2, the relationship between initial customer mixes and the frequency with which firm 1 dominates becomes flatter; meaning that the likelihood of dominance is less responsive to a firm's customer base. Figure 5 shows this more generally. The horizontal axis measures the degree of firm 1's first-mover advantage where it has no advantage at .5/.5 and, from that point upward, its advantage is increasing. As $\rho$ increases, the curve flattens which indicates that, for any initial advantage, the frequency with which firm 1 dominates is reduced. From these results, it is concluded that a higher rate of consumer experimentation weakens a first-mover advantage.

In contrast to the rate of consumer experimentation, Figure 6 shows that a higher rate of new ideas does complement a firm's first-mover advantage. As $\omega$ increases, the frequency with which firm 1 dominates, when it has a first-mover advantage, increases. When it has a superior customer mix, a higher rate of discovery makes it more likely that it'll identify and adopt a practice attractive to type 0 consumers before it loses its advantageous customer mix.

\section{Horizontally and Vertically Differentiated Prac- tices}

An unsatisfactory property of the previous model is that there is always market dominance in the long-run. Thus, the model has nothing to say about when we should expect to observe one firm sustaining a dominant position in the market. To address that issue, the previous model is enriched in the current section by allowing firms' practices to be both horizontally and vertically differentiated; that is, allowing the quality of practices to differ between firms.

\footnotetext{
${ }^{16}$ Movie 3 is especially revealing of this point.
} 
Let $z_{i}^{t}$ denote the quality of firm $i$ in period $t$. A type $k$ consumer prefers a firm with practices $x^{\prime}$ and quality $z^{\prime}$ to a firm with practices $x^{\prime \prime}$ and quality $z^{\prime \prime}$ iff

$$
\left|x^{\prime}-k\right|-\left|x^{\prime \prime}-k\right|<z^{\prime}-z^{\prime \prime}
$$

The equations of motion are then re-defined to be:

$$
\begin{aligned}
& \beta^{t+1}= \begin{cases}\beta^{t}+\rho\left(\alpha-\beta^{t}\right) & \text { if } x_{1}^{t-1}-x_{2}^{t}<z_{1}^{t-1}-z_{2}^{t} \text { and } x_{1}^{t}-x_{2}^{t-1}<z_{1}^{t}-z_{2}^{t-1} \\
\beta^{t} & \text { if } x_{1}^{t-1}-x_{2}^{t}<z_{1}^{t-1}-z_{2}^{t} \text { and } z_{1}^{t}-z_{2}^{t-1}<x_{1}^{t}-x_{2}^{t-1} \\
(1-\rho) \beta^{t}+\rho\left(\alpha-\beta^{t}\right) & \text { if } z_{1}^{t-1}-z_{2}^{t}<x_{1}^{t-1}-x_{2}^{t} \text { and } x_{1}^{t}-x_{2}^{t-1}<z_{1}^{t}-z_{2}^{t-1} \\
(1-\rho) \beta^{t} & \text { if } z_{1}^{t-1}-z_{2}^{t}<x_{1}^{t-1}-x_{2}^{t} \text { and } z_{1}^{t}-z_{2}^{t-1}<x_{1}^{t}-x_{2}^{t-1}\end{cases} \\
& \gamma^{t+1}= \begin{cases}\gamma^{t}+\rho\left(1-\alpha-\gamma^{t}\right) & \text { if } x_{2}^{t}-x_{1}^{t-1}<z_{1}^{t-1}-z_{2}^{t} \text { and } x_{2}^{t-1}-x_{1}^{t}<z_{1}^{t}-z_{2}^{t-1} \\
\gamma^{t} & \text { if } x_{2}^{t}-x_{1}^{t-1}<z_{1}^{t-1}-z_{2}^{t} \text { and } z_{1}^{t}-z_{2}^{t-1}<x_{2}^{t-1}-x_{1}^{t} \\
(1-\rho) \gamma^{t}+\rho\left(1-\alpha-\gamma^{t}\right) & \text { if } z_{1}^{t-1}-z_{2}^{t}<x_{2}^{t}-x_{1}^{t-1} \text { and } x_{2}^{t-1}-x_{1}^{t}<z_{1}^{t}-z_{2}^{t-1} \\
(1-\rho) \gamma^{t} & \text { if } z_{1}^{t-1}-z_{2}^{t}<x_{2}^{t}-x_{1}^{t-1} \text { and } z_{1}^{t}-z_{2}^{t-1}<x_{2}^{t-1}-x_{1}^{t}\end{cases}
\end{aligned}
$$

Assume $z_{i}^{t} \in \Delta$ where $\Delta$ is a finite subset of $[0, \bar{z}]$ and $\bar{z}>0$ and quality affects current profit in a proportional manner. This allows us to retain $\pi_{i}\left(x_{i}^{t}, \beta^{t}, \gamma^{t}\right)$ as a firm's profit function wlog. ${ }^{17}$ Note that one can interpret firms as receiving ideas that affect the vertical dimension (that is, $z_{i}^{t}$ ) as well as the horizontal dimension (that is, $x_{i}^{t}$ ). Since profit is monotonically increasing in quality, it'll always adopt quality-improving ideas. A state is now defined by $s^{t} \equiv\left(x_{1}^{t-1}, x_{2}^{t-1}, z_{1}^{t-1}, z_{2}^{t-1}, \beta^{t}, \gamma^{t}\right)$ and let $\Gamma$ denote the state space.

What is important for the analysis is the quality differential between firms, $z_{1}^{t}-z_{2}^{t}$. It is assumed to take on a finite number of values which lie in $\Psi$. Note that $\Psi$ is induced by $\Delta$ and is a subset of $[-\bar{z}, \bar{z}]$. Define $\mu: \Psi \times \Gamma \rightarrow[0,1]$ to be the probability function over $z_{1}^{t}-z_{2}^{t}$. Note that it can depend on the current state. ${ }^{18}$ A4 requires that positive probability be assigned to firms having identical qualities and to the extreme values. A5 requires that the probability that the quality differential does not change over a finite number of periods is positive.

A4 $\mu\left(\delta \mid s^{t}\right)>0 \forall \delta \in\{-\bar{z}, 0, \bar{z}\}, \forall s^{t} \in \Gamma$.

A5 If $\mu\left(\delta \mid s^{t}\right)>0$ then $\forall$ finite $T, \Pi_{\tau=t+1}^{t+T} \mu\left(\delta \mid s^{\tau}\right)>0 \forall s^{\tau}$ such that $z_{1}^{\tau-1}-z_{2}^{\tau-1}=\delta$.

Theorem 7 establishes that our earlier results are robust to allowing for vertical differentiation as long as the maximum quality differential is not too large.

Theorem 7 If $\bar{z} \in[0, \bar{\phi}-\underline{\phi})$ then, almost surely, $\lim _{t \rightarrow \infty}\left(\beta^{t}, \gamma^{t}\right) \in\{(\alpha, 0),(0,1-\alpha)\}$.

\footnotetext{
${ }^{17}$ That is, suppose profit is $h\left(z_{i}^{t}\right) \pi_{i}\left(x_{i}^{t}, \beta^{t}, \gamma^{t}\right)$ where $h(0)>0$ and $h^{\prime}\left(z_{i}^{t}\right)>0$. Then the adoption decisions regarding ideas are unaffected by the quality of a firm's practices.

${ }^{18}$ For example, it can allow for the probability that the quality differential is more likely to grow than to shrink which would be true if the quality leader has an advantage.
} 
With $\Gamma^{\prime}, \Gamma^{\prime \prime} \subset \Gamma$, let $\operatorname{Pr}\left(\Gamma^{\prime} \mid \Gamma^{\prime \prime}\right)$ denote the probability that $s^{t+\tau} \in \Gamma^{\prime}$ for some $\tau$, given $s^{t} \in \Gamma^{\prime \prime}$. Partition the state space into the following four sets:

$$
\begin{aligned}
\Gamma_{1} & \equiv\left\{s \in \Gamma: \beta>\frac{\alpha}{2}, \gamma<\frac{1-\alpha}{2}\right\}, \Gamma_{2} \equiv\left\{s \in \Gamma: \beta<\frac{\alpha}{2}, \gamma>\frac{1-\alpha}{2}\right\} \\
\Gamma_{3} & \equiv\left\{s \in \Gamma: \beta \geq \frac{\alpha}{2}, \gamma \geq \frac{1-\alpha}{2}\right\}, \Gamma_{4} \equiv\left\{s \in \Gamma: \beta \leq \frac{\alpha}{2}, \gamma \leq \frac{1-\alpha}{2}\right\}
\end{aligned}
$$

$\Gamma_{1}\left(\Gamma_{2}\right)$ is the set of states in which firm 1 (2) is dominating type 0 consumers (that is, its share of type 0 consumers exceeds .5) and firm 2 (1) is dominating type 1 consumers. $\Gamma_{3}\left(\Gamma_{4}\right)$ is the set of states in which firm 1 (2) is dominating both consumer types.

Theorem 8 shows that, if the maximum quality differential is sufficiently large then the state will ultimately be in each of these sets. Furthermore, since $\operatorname{Pr}\left(\Gamma_{i} \mid \Gamma_{i}\right)=1$ then $\Gamma_{i}$ is recurrent so the state will visit $\Gamma_{i}$ infinitely often. In other words, both firms dominate infinitely often with probability one so that market dominance is not sustained.

Theorem 8 If $\bar{z}>\bar{\phi}-\underline{\phi}$ then $\operatorname{Pr}\left(\Gamma_{i} \mid \Gamma_{j}\right)=1 \forall i, j \in\{1,2,3,4\}$.

To summarize, one firm will have a market share asymptotically approaching $(1-\rho) \alpha+\rho(1-\alpha)$ when the maximum quality differential is less than $\bar{\phi}-\underline{\phi}$. When instead the maximum quality differential exceeds $\bar{\phi}-\phi$, the identity of the market leader changes over time, even in the long-run. $\bar{\phi}-\phi \overline{\text { is }}$ then a critical value which determines whether or not there is sustained market dominance. It is straightforward to show that this critical value is smaller when consumers search at a higher rate. Using the first-order conditions defining $\underline{\phi}$ and $\bar{\phi}$, it can be derived:

$\frac{\partial \underline{\phi}}{\partial \rho}=\frac{\alpha g^{\prime}(\underline{\phi})+(1-\alpha) g^{\prime}(1-\underline{\phi})}{(1-\rho) \alpha g^{\prime \prime}(\underline{\phi})+\rho(1-\alpha) g^{\prime \prime}(1-\underline{\phi})}>0, \frac{\partial \bar{\phi}}{\partial \rho}=\frac{-\alpha g^{\prime}(\bar{\phi})-(1-\alpha) g^{\prime}(1-\bar{\phi})}{\rho \alpha g^{\prime \prime}(\bar{\phi})+(1-\rho)(1-\alpha) g^{\prime \prime}(1-\bar{\phi})}<0 ;$

so that $\bar{\phi}-\phi$ is decreasing in $\rho$. Therefore, sustained market dominance is less likely when consumers experiment at a higher rate.

To understand this result, one must first recognize that the crucial issue regarding sustained market dominance is whether firms' locations can be sufficiently far apart in the long-run so that even if the market laggard has higher quality, it does not alter consumers' loyalty decisions. When firms have comparable qualities and, say, firm 1 is dominant then firms' optimal location decisions are converging to $\phi$ for firm 1 and $\bar{\phi}$ for firm 2. Their practices will probabilistically converge to those locations. Therefore, in finite time with positive probability, $\left|x_{1}^{t}-x_{2}^{t}\right|$ is close to $\bar{\phi}-\phi$. If $\bar{z}<\bar{\phi}-\phi$, so that $\bar{z}<\left|x_{1}^{t}-x_{2}^{t}\right|$, then firm 2 cannot induce type 0 customers to become loyal to it even when it has higher quality practices. This is the basis for Theorem 7 . However, if $\bar{z}>\bar{\phi}-\underline{\phi}$ then higher quality induces type 0 customers to become loyal to firm 
2 and, in fact, both consumer types are attracted to it. ${ }^{19}$ The role of the rate of consumer experimentation, $\rho$, is as follows. By raising $\rho$, firms' have more similar mixes of consumers buying from them as, in any period, there is a larger fraction of "noise" consumers; consumers who, in that period, are choosing a firm irrespective of their loyalty. The increased similarity in customer bases causes the long-run value for $\left|x_{1}^{t}-x_{2}^{t}\right|$ to shrink. As a result, it becomes more likely that a quality advantage can cause consumers to switch loyalties and turn a market laggard into a market leader.

\section{Concluding Remarks}

If, as firms' locations settled down, consumer experimentation went to zero, it would not be surprising if sustained market dominance prevailed. Even if a non-dominant firm adopted a location that would be attractive to the prevalent consumer type, there would be little consumer response to it. If firms engaged in local search - trying to find profit-improving ideas around their current location - it would also not be surprising if sustained dominance emerged. There might be ideas that would allow a non-dominant firm to become dominant but a firm would never find them because of its localized search. Finally, if firms faced a cost to adjusting their location, it would once again not be surprising that sustained market dominance would emerge. What is striking about our analysis is that - in spite of consumers always engaging in experimentation, firms always engaging in global search, and firms being able to costlessly adjust their locations - sustained market dominance must still prevail. Furthermore, this result is robust to the rate of consumer experimentation though it is not robust to allowing for sufficiently great shocks to the quality differential between firms.

Obviously, a serious restriction in our analysis is that firms are myopic optimizers. Specifically, it is assumed that new practices are adopted if and only if they raise profit based on the current set of customers. A virtue to this rule is that it is sensible it'll always lead to short-run profit increases - and it is feasible - it requires minimal information and sophistication on the part of the firms. The other extreme would be to characterize a Markov perfect equilibrium with firms valuing the discounted stream of expected profits. The appeal there is that firms can take into account how their practices affect their future customer bases. The obvious disadvantage is that it is not at all clear that such strategies are feasible because: i) there may not exist a plausible learning dynamic that converges to Markov equilibrium strategies; or ii) it may not be computationally feasible for firms to solve for a Markov equilibrium strategy. ${ }^{20}$ The hope for future research is to find middle ground between these two

\footnotetext{
${ }^{19}$ Interestingly, when one firm is preferred by both consumer types then firms' locations probabilistically converge to the same value, $\widehat{\phi}$ (see footnote 11 ).

${ }^{20}$ Though see Pakes (2000) for a discussion of a stochastic algorithm for learning Markov equilibrium strategies.
} 
extreme behavioral assumptions by identifying heuristics that take into account the relationship between practices and customer loyalty decisions.

A second serious restriction is that price-setting behavior is not modelled. If prices are set to maximize static profit then allowing firms to choose prices should not upset results. Since, in the absence of price-setting, firms' practices tend to diverge, allowing firms to choose price should reinforce that tendency since more similar practices result in more intense price competition. More interesting is if firms are forward-looking and use price to attract consumers with the intent that they may become loyal customers. A non-dominant firm could potentially displace the dominant firm by pricing sufficiently low since a price differential is equivalent to a quality differential and we know that market dominance is not sustained if the quality differential is sufficiently large. Determining optimal pricing in that context would be quite interesting. 


\section{Appendix}

Proof of Lemma 1: Let us first show that if $\pi$ has an optimum, it is an interior solution. Consider $x=0$ :

$$
\frac{\partial \pi(0, w(0), w(1))}{\partial x}=-[(1-\rho) w(1)+\rho(1-\alpha-w(1))] g^{\prime}(1)>0
$$

since $g^{\prime}(0)=0$. Hence, if $\phi$ exists then $\phi>0$. Next consider:

$$
\frac{\partial \pi(1, w(0), w(1))}{\partial x}=[(1-\rho) w(0)+\rho(\alpha-w(0))] g^{\prime}(1)<0
$$

Hence, if $\phi$ exists then $\phi<1$. Given an optimum must be interior and $\pi$ is strictly concave then $\phi$ is defined by the first-order condition:

$$
\begin{aligned}
& {[(1-\rho) w(0)+\rho(\alpha-w(0))] g^{\prime}(\phi)} \\
& -[(1-\rho) w(1)+\rho(1-\alpha-w(1))] g^{\prime}(1-\phi)=0
\end{aligned}
$$

Define

$$
\begin{aligned}
\Delta \equiv & {[(1-\rho) w(0)+\rho(\alpha-w(0))] g^{\prime \prime}(\phi) } \\
& +[(1-\rho) w(1)+\rho(1-\alpha-w(1))] g^{\prime \prime}(1-\phi)<0,
\end{aligned}
$$

as $g$ is strictly concave. Taking the total derivative of (13) with respect to $w(0)$, one finds:

$$
\frac{\partial \phi}{\partial w(0)}=\frac{(2 \rho-1) g^{\prime}(\phi)}{\Delta}<0
$$

since $\rho \in(0,1 / 2)$. Similarly,

$$
\frac{\partial \phi}{\partial w(1)}=\frac{(1-2 \rho) g^{\prime}(1-\phi)}{\Delta}>0
$$

For notational ease, let $\phi_{1}^{t} \equiv \phi_{1}\left(\beta^{t}, \gamma^{t}\right), \phi_{2}^{t} \equiv \phi_{2}\left(\beta^{t}, \gamma^{t}\right), \psi_{1}^{t} \equiv \psi_{1}\left(x_{1}^{t-1}, \beta^{t}, \gamma^{t}\right)$, and $\psi_{2}^{t} \equiv \psi_{2}\left(x_{2}^{t-1}, \beta^{t}, \gamma^{t}\right)$.

Proof of Lemma 2: This is trivially true if $\widetilde{\beta}=\bar{\beta}$ and $\widetilde{\gamma}=\bar{\gamma}$ so, from hereon, it is assumed that $\widetilde{\beta}>\bar{\beta}$ and/or $\widetilde{\gamma}<\bar{\gamma}$. Let $\widetilde{\phi}_{i}^{t}$ and $\bar{\phi}_{i}^{t}$ be the optimal locations for firm $i$ when the state variables are $(\widetilde{\beta}, \widetilde{\gamma})$ and $(\bar{\beta}, \bar{\gamma})$, respectively. From Lemma 1 it follows that $\widetilde{\phi}_{1}^{t}<\bar{\phi}_{1}^{t}$ and $\bar{\phi}_{2}^{t}<\widetilde{\phi}_{2}^{t}$. Let $\widetilde{H}_{i}$ and $\bar{H}_{i}$ denote the cdf on $x_{i}^{t}$ when the period $t-1$ state variables are $(\widetilde{\beta}, \widetilde{\gamma})$ and $(\bar{\beta}, \bar{\gamma})$, respectively. The result will only be shown for firm 1 as the proof for firm 2 is analogous.

Suppose $\left(\psi_{1}^{t}<\right) \phi_{1}^{t}<x_{1}^{t-1}$. Given a location of $x_{1}^{t-1}$, firm 1 will adopt the period $t$ idea, $y_{1}^{t}$, iff $y_{1}^{t} \in\left[\psi_{1}^{t}, x_{1}^{t-1}\right]$. The probability that $x_{1}^{t}$ lies in a subset of $\left[\psi_{1}^{t}, x_{1}^{t-1}\right)$ is the probability of firm 1 receiving a new idea, which is $\omega$, times the probability of $y_{1}^{t}$ 
lying in that subset. The residual probability is assigned to $x_{1}^{t}=x_{1}^{t-1}$. Since $F$ is the cdf on $y_{1}^{t}$, we then have

$$
H_{1}\left(x_{1}^{t}\right)= \begin{cases}0 & \text { if } x_{1}^{t} \leq \psi_{1}^{t} \\ \omega\left[F\left(x_{1}^{t}\right)-F\left(\psi_{1}^{1}\right)\right] & \text { if } \psi_{1}^{1} \leq x_{1}^{t}<x_{1}^{t-1} \\ 1 & \text { if } x_{1}^{t-1} \leq x_{1}^{t}\end{cases}
$$

Suppose $x_{1}^{t-1}<\phi_{1}^{t}\left(<\psi_{1}^{t}\right)$. Then

$$
H_{1}\left(x_{1}^{t}\right)= \begin{cases}0 & \text { if } x_{1}^{t}<x_{1}^{t-1} \\ (1-\omega)+\omega\left[F\left(x_{1}^{t-1}\right)+1-F\left(\psi_{1}^{t}\right)\right] & \text { if } x_{1}^{t}=x_{1}^{t-1} \\ (1-\omega)+\omega\left[F\left(x_{1}^{t-1}\right)+1-F\left(\psi_{1}^{t}\right)\right]+\omega\left[F\left(x_{1}^{t}\right)-F\left(x_{1}^{t-1}\right)\right] & \text { if } x_{1}^{t-1} \leq x_{1}^{t} \leq \psi_{1}^{t} \\ 1 & \text { if } \psi_{1}^{t} \leq x_{1}^{t} .\end{cases}
$$

There are three generic cases to consider: i) $\widetilde{\phi}_{1}^{t}<\bar{\phi}_{1}^{t}<x_{1}^{t-1}$; ii) $\widetilde{\phi}_{1}^{t}<x_{1}^{t-1}<\bar{\phi}_{1}^{t}$; and iii) $x_{1}^{t-1}<\widetilde{\phi}_{1}^{t}<\bar{\phi}_{1}^{t}$. For each case, we'll show that $\bar{H}_{1}$ stochastically dominates $\widetilde{H}_{1}$ in the first degree; that is,

$$
\bar{H}_{1}\left(x_{1}^{t}\right) \leq \widetilde{H}_{1}\left(x_{1}^{t}\right) \forall x_{1}^{t} .
$$

i) Assume $\widetilde{\phi}_{1}^{t}<\bar{\phi}_{1}^{t}<x_{1}^{t-1}$. The plan is to show that $\widetilde{\psi}_{1}^{t} \leq \bar{\psi}_{1}^{t}$. When $\psi_{1}^{t}$ is in the interior of $[0,1]$, it is defined by: $\pi\left(\psi_{1}^{t}\left(x_{1}^{t-1}, \beta^{t}, \gamma^{t}\right), \beta^{t}, \gamma^{t}\right)=\pi\left(x_{1}^{t-1}, \beta^{t}, \gamma^{t}\right)$. Taking the total derivative with respect to $\beta^{t}$, we derive:

$$
\frac{\partial \psi_{1}^{t}}{\partial \beta^{t}}=\left[\frac{\partial \pi\left(x_{1}^{t-1}, \beta^{t}, \gamma^{t}\right)}{\partial \beta^{t}}-\frac{\partial \pi\left(\psi_{1}^{t}, \beta^{t}, \gamma^{t}\right)}{\partial \beta^{t}}\right] / \frac{\partial \pi\left(\psi_{1}^{t}, \beta^{t}, \gamma^{t}\right)}{\partial x_{1}^{t-1}}
$$

If $\psi_{1}^{t}<\phi_{1}^{t}<x_{1}^{t-1}$ then $\partial \pi\left(\psi_{1}^{t}, \beta^{t}, \gamma^{t}\right) / \partial x_{1}^{t-1}>0$ and $\partial \pi\left(x_{1}^{t-1}, \beta^{t}, \gamma^{t}\right) / \partial \beta^{t}<$ $\partial \pi\left(\psi_{1}^{t}, \beta^{t}, \gamma^{t}\right) / \partial \beta^{t}$ which follows from $\partial^{2} \pi / \partial x_{1}^{t-1} \partial \beta^{t}<0$. Hence, if $\phi_{1}^{t}<x_{1}^{t-1}$ then $\partial \psi_{1}^{t} / \partial \beta^{t}<0$. By an analogous argument, one can show that $\partial \psi_{1}^{t} / \partial \gamma^{t}>0$. Since $\widetilde{\beta} \geq \bar{\beta}$ and $\widetilde{\gamma} \leq \bar{\gamma}$ then: i) if $\bar{\psi}_{1}^{t}=0$ then $\widetilde{\psi}_{1}^{t}=0$; and ii) if $\bar{\psi}_{1}^{t}>0$ then $\widetilde{\psi}_{1}^{t}<\bar{\psi}_{1}^{t}$. Therefore, $\widetilde{\psi}_{1}^{t} \leq \bar{\psi}_{1}^{t}$. It follows that

$$
\widetilde{H}_{1}\left(x_{1}^{t}\right)-\bar{H}_{1}\left(x_{1}^{t}\right)= \begin{cases}0 & \text { if } x_{1}^{t} \leq \widetilde{\psi}_{1}^{t} \\ \omega\left[F\left(x_{1}^{t}\right)-F\left(\widetilde{\psi}_{1}^{t}\right)\right] & \text { if } \widetilde{\psi}_{1}^{t} \leq x_{1}^{t} \leq \bar{\psi}_{1}^{t} \\ \omega\left[F\left(\bar{\psi}_{1}^{t}\right)-F\left(\widetilde{\psi}_{1}^{t}\right)\right] & \text { if } \bar{\psi}_{1}^{t} \leq x_{1}^{t} \leq x_{1}^{t-1} \\ 0 & \text { if } x_{1}^{t-1} \leq x_{1}^{t}\end{cases}
$$

so that (17) holds. 
ii) Assume $\left(\widetilde{\psi}_{1}^{t}<\right) \widetilde{\phi}_{1}^{t}<x_{1}^{t-1}<\bar{\phi}_{1}^{t}\left(<\bar{\psi}_{1}^{t}\right)$. It follows that

$$
\widetilde{H}_{1}\left(x_{1}^{t}\right)-\bar{H}_{1}\left(x_{1}^{t}\right)= \begin{cases}0 & \text { if } x_{1}^{t} \leq \widetilde{\psi}_{1}^{t} \\ \omega\left[F\left(x_{1}^{t}\right)-F\left(\widetilde{\psi}_{1}^{t}\right)\right] & \text { if } \widetilde{\psi}_{1}^{t} \leq x_{1}^{t}<x_{1}^{t-1} \\ \omega\left[F\left(\bar{\psi}_{1}^{t}\right)-F\left(x_{1}^{t-1}\right)\right] & \text { if } x_{1}^{t}=x_{1}^{t-1} \\ \omega\left[F\left(\bar{\psi}_{1}^{t}\right)-F\left(x_{1}^{t}\right)\right] & \text { if } x_{1}^{t-1} \leq x_{1}^{t} \leq \bar{\psi}_{1}^{t} \\ 0 & \text { if } \bar{\psi}_{1}^{t} \leq x_{1}^{t}\end{cases}
$$

so that (17) holds.

iii) Assume $x_{1}^{t-1}<\widetilde{\phi}_{1}^{t}<\bar{\phi}_{1}^{t}$. By an analogous argument to that used in (i), we can show $\widetilde{\psi}_{1}^{t} \leq \bar{\psi}_{1}^{t}$ and, therefore, $x_{1}^{t-1}<\widetilde{\psi}_{1}^{t} \leq \bar{\psi}_{1}^{t}$. It follows that

$$
\widetilde{H}_{1}\left(x_{1}^{t}\right)-\bar{H}_{1}\left(x_{1}^{t}\right)= \begin{cases}0 & \text { if } x_{1}^{t} \leq x_{1}^{t-1} \\ \omega\left[F\left(\bar{\psi}_{1}^{t}\right)-F\left(\widetilde{\psi}_{1}^{t}\right)\right] & \text { if } x_{1}^{t-1} \leq x_{1}^{t} \leq \widetilde{\psi}_{1}^{t} \\ \omega\left[F\left(\bar{\psi}_{1}^{t}\right)-F\left(x_{1}^{t}\right)\right] & \text { if } \widetilde{\psi}_{1}^{t} \leq x_{1}^{t} \leq \bar{\psi}_{1}^{t} \\ 0 & \text { if } \bar{\psi}_{1}^{t} \leq x_{1}^{t}\end{cases}
$$

so that (17) holds.

This establishes that (17) is true. Since $\bar{\phi}_{2}^{t}<\widetilde{\phi}_{2}^{t}$, by an analogous argument one can show that $\widetilde{H}_{2}$ stochastically dominates $\bar{H}_{2}$ in the first degree.

Proof of Theorem 3: There are two possible outcomes: i) $x_{1} \neq x_{2}$ so that one firm's loyal customers are type 0 and the other firm's are type 1 ; and ii) $x_{1}=x_{2}$ so that each firm serves half of each consumer type. Let us first show that there does not exist an equilibrium with $x_{1} \neq x_{2}$. Wlog, suppose $x_{1}<x_{2}$ so that all type 0 consumers prefer to buy from firm 1 and all type 1 consumers prefer to buy from firm 2. It is immediate that $\left(x_{1}, x_{2}\right)=(\underline{\phi}, \bar{\phi})$ and firms' payoffs are:

$$
\begin{aligned}
& \pi_{1}(\alpha) \equiv(1-\rho) \alpha g(\underline{\phi})+\rho(1-\alpha) g(1-\underline{\phi}) \\
& \pi_{2}(\alpha) \equiv \rho \alpha g(\bar{\phi})+(1-\rho)(1-\alpha) g(1-\bar{\phi}) ;
\end{aligned}
$$

where, for later analysis, the dependence on $\alpha$ is made explicit. Also note that $\underline{\phi}$ and $\bar{\phi}$ are both functions of $\alpha$. As an alternative to $\bar{\phi}$, firm 2 could locate below but arbitrarily close to $\phi$ and earn $\pi_{1}(\alpha)$. Hence, a necessary condition for $(\phi, \bar{\phi})$ to be an equilibrium is that $\pi_{1}(\alpha) \leq \pi_{2}(\alpha)$. Analogously, firm 1 must prefer locating at $\underline{\phi}$ to locating above but arbitrarily close to $\bar{\phi}$ and earning $\pi_{2}(\alpha)$. Equilibrium then requires $\pi_{1}(\alpha) \geq \pi_{2}(\alpha)$. Combining these two conditions, equilibrium requires $\pi_{1}(\alpha)=\pi_{2}(\alpha)$. The next step is to show that if $\alpha>1 / 2$ that this condition cannot hold and, more specifically, $\pi_{1}(\alpha)>\pi_{2}(\alpha)$. 
First note that $\pi_{1}(1 / 2)=\pi_{2}(1 / 2)$. Next, let us evaluate the change in these profit functions with respect to $\alpha$.

$$
\begin{aligned}
\pi_{1}^{\prime}(\alpha)= & (1-\rho) g(\underline{\phi})-\rho g(1-\underline{\phi}) \\
& +\underline{\phi}^{\prime}(\alpha)\left[(1-\rho) \alpha g^{\prime}\left(\underline{\phi}-\rho(1-\alpha) g^{\prime}(1-\underline{\phi})\right]\right. \\
= & (1-\rho) g(\underline{\phi}-\rho g(1-\underline{\phi})
\end{aligned}
$$

as $(1-\rho) \alpha g^{\prime}(\underline{\phi})-\rho(1-\alpha) g^{\prime}(1-\underline{\phi})=0$ is the first-order condition defining $\underline{\phi}$. Since $(1-\rho) \alpha>\rho(1-\alpha)$ then the first-order condition implies $g^{\prime}(\phi)>g^{\prime}(1-\bar{\phi})$ (where recall that $g^{\prime}<0$ ). Given $g^{\prime \prime}<0$ then this implies $\underline{\phi}<1-\underline{\phi}$ and thus $\underline{\phi}<1 / 2$. It follows that $g(\underline{\phi})>g(1-\underline{\phi})$ and hence $\pi_{1}^{\prime}(\alpha)>0$. Next consider:

$$
\pi_{2}^{\prime}(\alpha)=\rho g(\bar{\phi})-(1-\rho) g(1-\bar{\phi}) \text {. }
$$

Since $\bar{\phi}>1 / 2$ when $\alpha=1 / 2$ then $\pi_{2}^{\prime}(1 / 2)<0$. For $\alpha$ near $1 / 2$, it follows that $\pi_{1}$ is increasing in $\alpha$ and $\pi_{2}$ is decreasing in $\alpha$. Hence, if $\exists \alpha \in(1 / 2,1)$ such that $\pi_{1}(\alpha)=\pi_{2}(\alpha)$ then, by these functions being continuously differentiable with respect to $\alpha, \exists \alpha^{o}$ such that $\pi_{1}\left(\alpha^{o}\right)=\pi_{2}\left(\alpha^{o}\right)$ and $\pi_{1}^{\prime}\left(\alpha^{o}\right) \leq \pi_{2}^{\prime}\left(\alpha^{o}\right)$ (that is, as $\alpha$ increases toward $\alpha^{o}, \pi_{2}$ approaches $\pi_{1}$ from below). $\pi_{1}^{\prime}\left(\alpha^{o}\right) \leq \pi_{2}^{\prime}\left(\alpha^{o}\right)$ iff

$$
\begin{gathered}
\rho g(\bar{\phi})-(1-\rho) g(1-\bar{\phi}) \geq(1-\rho) g(\underline{\phi})-\rho g(1-\underline{\phi}) \Leftrightarrow \\
\rho[g(1-\underline{\phi})+g(\bar{\phi})] \geq(1-\rho)[g(\underline{\phi})+g(1-\bar{\phi})] .
\end{gathered}
$$

Recall that, by Lemma $1, \underline{\phi}<\bar{\phi}$. Since $g(\underline{\phi})>g(1-\underline{\phi})$, as $\underline{\phi}<1 / 2$, and $1-\rho>\rho$, a necessary condition for $(18)$ to hold is that $g(\bar{\phi})>g(1-\bar{\phi})$ which implies $\bar{\phi}<1 / 2$. Given $\phi<\bar{\phi}<1 / 2$, note that firm 1's profit from setting $x_{1}$ below but arbitrarily close to $\bar{\phi}$ is $(1-\rho) \alpha^{o} g(\bar{\phi})+\rho\left(1-\alpha^{o}\right) g(1-\bar{\phi})$ which exceeds $\pi_{2}\left(\alpha^{o}\right)$. Therefore, $\pi_{1}\left(\alpha^{o}\right)>\pi_{2}\left(\alpha^{o}\right)$. This shows $\nexists \alpha$ such that $\pi_{1}(\alpha)=\pi_{2}(\alpha)$ and hence there does not exist a Nash equilibrium in which $x_{1} \neq x_{2}$.

Now consider $\left(x_{1}, x_{2}\right)=(x, x)$. Each firm's payoff is $(\alpha / 2) g(x)+[(1-\alpha) / 2] g(1-x)$. Suppose $x \in(0,1)$. A necessary condition for equilibrium is that locating at $x$ is preferable to locating below but arbitrarily close to $x$ :

$$
\begin{aligned}
(\alpha / 2) g(x)+[(1-\alpha) / 2] g(1-x) & \geq(1-\rho) \alpha g(x)+\rho(1-\alpha) g(1-x) \Leftrightarrow \\
(1-\alpha) g(1-x) & \geq \alpha g(x) .
\end{aligned}
$$

Another necessary condition is that locating at $x$ is preferable to locating above but arbitrarily close to $x$ :

$$
\begin{aligned}
(\alpha / 2) g(x)+[(1-\alpha) / 2] g(1-x) & \geq \rho \alpha g(x)+(1-\rho)(1-\alpha) g(1-x) \Leftrightarrow \\
\alpha g(x) & \geq(1-\alpha) g(1-x) .
\end{aligned}
$$


Combining these two conditions yields: $\alpha g(x)=(1-\alpha) g(1-x)$. At a value of $x$ that satisfies that equality, a firm is indifferent between locating at $x$ and locating arbitrarily below $x$ (and focusing on type 0 consumers) and arbitrarily above $x$ (and focusing on type 1 consumers). If $\phi<x$ then locating at $\phi$ is strictly preferred to locating below and arbitrarily close to $x$. In that case, locating at $\underline{\phi}$ is strictly preferred to locating at $x$. It is then necessary that $x \leq \phi$. By the same logic, it is necessary that $\bar{\phi} \leq x$. As this implies $x \leq \underline{\phi}<\bar{\phi} \leq x$, it is concluded that $\nexists x \in(0,1)$ such that $(x, x)$ is an equilibrium.

Consider $x=1$. Each firm's payoff is $(\alpha / 2) g(1)+[(1-\alpha) / 2] g(0)$ and this is strictly less than locating at 0 and earning $(1-\rho) \alpha g(0)+\rho(1-\alpha) g(1)$. So $(1,1)$ is not an equilibrium.

Finally, consider $x=0$. The necessary and sufficient condition for equilibrium is that a firm prefers to locate at 0 , and share both consumer types, than to locate at $\bar{\phi}$ and serve only type 1 consumers. This holds iff $\Psi(\alpha) \geq 0$ where

$$
\begin{aligned}
\Psi(\alpha) \equiv & (\alpha / 2) g(0)+((1-\alpha) / 2) g(1) \\
& -\rho \alpha g(\bar{\phi})-(1-\rho)(1-\alpha) g(1-\bar{\phi}) .
\end{aligned}
$$

When $\alpha=1 / 2$, a firm strictly prefers to locate at 1 given the other firm is at 0 iff

$$
(1-\rho)(1 / 2) g(0)+\rho(1 / 2) g(1)>(1 / 4) g(0)+(1 / 4) g(1)
$$

which is indeed true. Hence, $\Psi(1 / 2)<0$. Next note that it is an equilibrium for both firms to locate at 0 when $\alpha=1: \Psi(1)=(1 / 2) g(0)-\rho g(\bar{\phi})>0$. To conclude the proof, let us show that if $\Psi(\alpha)=0$ then $\Psi^{\prime}(\alpha)>0$. By $\Psi$ being continuously differentiable, this implies that there is a unique value for $\alpha$ such that $\Psi(\alpha)=0$.

$$
\begin{aligned}
\Psi^{\prime}(\alpha)= & (1 / 2)[g(0)-g(1)]-[\rho g(\bar{\phi})-(1-\rho) g(1-\bar{\phi})] \\
& -\bar{\phi}^{\prime}(\alpha)\left[\rho \alpha g^{\prime}(\bar{\phi})-(1-\rho)(1-\alpha) g^{\prime}(1-\bar{\phi})\right] .
\end{aligned}
$$

Note that $\rho \alpha g^{\prime}(\bar{\phi})-(1-\rho)(1-\alpha) g^{\prime}(1-\bar{\phi})=0$ by the first-order condition defining $\bar{\phi}$. It follows that:

$$
\Psi^{\prime}(\alpha)=(1 / 2)[g(0)-g(1)]-[\rho g(\bar{\phi})-(1-\rho) g(1-\bar{\phi})] .
$$

Next note that $\Psi(\alpha)=0$ can be rearranged to yield

$$
(1 / 2)[g(0)-g(1)]-[\rho g(\bar{\phi})-(1-\rho) g(1-\bar{\phi})]=(1 / \alpha)[(1-\rho) g(1-\bar{\phi})-(1 / 2) g(1)] \text {. }
$$

Substituting this into (19):

$$
\Psi^{\prime}(\alpha)=(1 / \alpha)[(1-\rho) g(1-\bar{\phi})-(1 / 2) g(1)]>0
$$

as $g(1-\bar{\phi}) \geq g(1)$ and $1-\rho>1 / 2$. 
To summarize, $\left(x_{1}, x_{2}\right)=(0,0)$ is a Nash equilibrium iff $\Psi(\alpha) \geq 0$. It's been shown that $\Psi(1 / 2)<0<\Psi(1)$ and if $\Psi(\alpha)=0$ then $\Psi^{\prime}(\alpha)>0$. There then exists a unique value of $\alpha$ over $(1 / 2,1)$, denoted $\underline{\alpha}$, such that $\Psi(\alpha) \geq 0$ iff $\alpha \in[\underline{\alpha}, 1]$.

Proof of Theorem 4: Only the proof for when $\left(x_{1}^{t-1}, x_{2}^{t-1}, \beta^{t}, \gamma^{t}\right) \in \Omega_{1}$ is provided as the proof for when the state lies in $\Omega_{2}$ is the same.

\section{Lemma 9 If}

$$
\max \left\{\psi_{1}\left(x_{1}^{t-1}, \beta^{t}, \gamma^{t}\right), x_{1}^{t-1}\right\}<\min \left\{\psi_{2}\left(x_{2}^{t-1}, \beta^{t}, \gamma^{t}\right), x_{2}^{t-1}\right\}
$$

then with probability one,

$$
\begin{gathered}
\left(\beta^{t+1}, \gamma^{t+1}\right)=\left(\beta^{t}+\rho\left(\alpha-\beta^{t}\right),(1-\rho) \gamma^{t}\right) \\
\phi_{1}\left(\beta^{t+1}, \gamma^{t+1}\right)<\phi_{1}\left(\beta^{t}, \gamma^{t}\right), \text { and } \phi_{2}\left(\beta^{t}, \gamma^{t}\right)<\phi_{2}\left(\beta^{t+1}, \gamma^{t+1}\right) .
\end{gathered}
$$

Proof: Suppose $\phi_{1}^{t} \leq x_{1}^{t-1}$. It follows that $\psi_{1}^{t} \leq \phi_{1}^{t} \leq x_{1}^{t-1}$. Recall that $y_{1}^{t}$ is the new idea for period $t$ and is adopted iff it results in higher profit which, by the definition of $\psi_{1}$, means that $y_{1}^{t} \in\left[\psi_{1}^{t}, x_{1}^{t-1}\right)$. Firm 1 either adopts $y_{1}^{t}$ as its new location or maintains its current location. Therefore, its period $t$ location either lies in $\left[\psi_{1}^{t}, x_{1}^{t-1}\right]$ so that $x_{1}^{t} \leq x_{1}^{t-1}$ and thus $x_{1}^{t} \leq \max \left\{\psi_{1}^{t}, x_{1}^{t-1}\right\}$. Next suppose $x_{1}^{t-1}<\phi_{1}^{t}$. This implies $x_{1}^{t-1}<\phi_{1}^{t}<\psi_{1}^{t}$. Since $y_{1}^{t}$ is adopted iff $y_{1}^{t} \in\left(x_{1}^{t-1}, \psi_{1}^{t}\right]$, it follows that $x_{1}^{t} \leq \psi_{1}^{t}$. Hence, $x_{1}^{t} \leq \max \left\{\psi_{1}^{t}, x_{1}^{t-1}\right\}$. It follows that $x_{1}^{t} \leq \max \left\{\psi_{1}^{t}, x_{1}^{t-1}\right\}$. By an analogous argument, we can show that $\min \left\{\psi_{2}^{t}, x_{2}^{t-1}\right\} \leq x_{2}^{t}$.

We then have

$$
x_{1}^{t} \leq \max \left\{\psi_{1}^{t}, x_{1}^{t-1}\right\}<\min \left\{\psi_{2}^{t}, x_{2}^{t-1}\right\} \leq x_{2}^{t-1}
$$

so that $x_{1}^{t}<x_{2}^{t-1}$ and

$$
x_{1}^{t-1} \leq \max \left\{\psi_{1}^{t}, x_{1}^{t-1}\right\}<\min \left\{\psi_{2}^{t}, x_{2}^{t-1}\right\} \leq x_{2}^{t}
$$

so that $x_{1}^{t-1}<x_{2}^{t}$. It follows that (21) is true. Since $\phi$ is decreasing in $\beta$ and increasing in $\gamma,(22)$ is true by Lemma 1 .

Lemma 10 If

$$
\max \left\{\psi_{1}\left(x_{1}^{t-1}, \beta^{t}, \gamma^{t}\right), x_{1}^{t-1}\right\}<\min \left\{\psi_{2}\left(x_{2}^{t-1}, \beta^{t}, \gamma^{t}\right), x_{2}^{t-1}\right\}
$$

then

$$
\max \left\{\psi_{1}\left(x_{1}^{\tau}, \beta^{\tau+1}, \gamma^{\tau+1}\right), x_{1}^{\tau}\right\}<\min \left\{\psi_{2}\left(x_{2}^{\tau}, \beta^{\tau+1}, \gamma^{\tau+1}\right), x_{2}^{\tau}\right\}
$$

$\forall \tau \geq t$ 
Proof: (23) implies $x_{1}^{t-1}<x_{2}^{t-1}$. The proof strategy is to show that if (23) holds then

$$
\max \left\{\psi_{1}\left(x_{1}^{t}, \beta^{t+1}, \gamma^{t+1}\right), x_{1}^{t}\right\} \leq \max \left\{\psi_{1}\left(x_{1}^{t-1}, \beta^{t}, \gamma^{t}\right), x_{1}^{t-1}\right\} .
$$

We will then claim that, by an analogous argument, one can show that if (23) holds then

$$
\min \left\{\psi_{2}\left(x_{2}^{t-1}, \beta^{t}, \gamma^{t}\right), x_{2}^{t-1}\right\} \leq \min \left\{\psi_{2}\left(x_{2}^{t}, \beta^{t+1}, \gamma^{t+1}\right), x_{2}^{t}\right\} .
$$

Lemma 10 follows by induction.

i) Suppose $\phi_{1}^{t} \leq x_{1}^{t-1}$ and thus $\psi_{1}^{t} \leq \phi_{1}^{t} \leq x_{1}^{t-1}$. Partition the possible values for $x_{1}^{t}$ into $\left[\psi_{1}^{t}, \phi_{1}^{t+1}\right) \cup\left[\phi_{1}^{t+1}, x_{1}^{t-1}\right]$ where, from Lemma 9, we know that $\phi_{1}^{t+1}<\phi_{1}^{t} \cdot{ }^{21}$

i-a) Suppose $x_{1}^{t} \in\left[\phi_{1}^{t+1}, x_{1}^{t-1}\right]$. First note that $\phi_{1}^{t+1} \leq x_{1}^{t}$ implies $\psi_{1}^{t+1} \leq \phi_{1}^{t+1} \leq$ $x_{1}^{t}$. Therefore, $\max \left\{\psi_{1}^{t+1}, x_{1}^{t}\right\}=x_{1}^{t}$. Given that $\psi_{1}^{t} \leq x_{1}^{t-1}$ (as case (i) assumes $\phi_{1}^{t} \leq x_{1}^{t-1}$ ), we can conclude from $x_{1}^{t} \leq x_{1}^{t-1}$ that

$$
\left(x_{1}^{t}=\right) \max \left\{\psi_{1}^{t+1}, x_{1}^{t}\right\} \leq \max \left\{\psi_{1}^{t}, x_{1}^{t-1}\right\}\left(=x_{1}^{t-1}\right) .
$$

This proves (25) for when $\phi_{1}^{t} \leq x_{1}^{t-1}$ and $x_{1}^{t} \in\left[\phi_{1}^{t+1}, x_{1}^{t-1}\right]$.

i-b) Now suppose $x_{1}^{t} \in\left[\psi_{1}^{t}, \phi_{1}^{t+1}\right)$. We then have $x_{1}^{t}<\phi_{1}^{t+1}<\psi_{1}^{t+1}$. We want to prove that $\psi_{1}^{t+1}<x_{1}^{t-1}$. Suppose not so that $x_{1}^{t-1} \leq \psi_{1}^{t+1}$. Since, by the definition of $\psi_{1}^{t+1}$, any location over $\left(x_{1}^{t}, \psi_{1}^{t+1}\right]$ yields weakly higher profit than $x_{1}^{t}$ and it is postulated that $x_{1}^{t-1} \in\left(x_{1}^{t}, \psi_{1}^{t+1}\right]$, it follows that $\pi_{1}\left(x_{1}^{t-1}, \beta^{t+1}, \gamma^{t+1}\right) \geq \pi_{1}\left(x_{1}^{t}, \beta^{t+1}, \gamma^{t+1}\right)$. We also know that $\pi_{1}\left(x_{1}^{t-1}, \beta^{t}, \gamma^{t}\right) \leq$ $\pi_{1}\left(x_{1}^{t}, \beta^{t}, \gamma^{t}\right)$. We then have

$$
\pi_{1}\left(x_{1}^{t}, \beta^{t}, \gamma^{t}\right)-\pi_{1}\left(x_{1}^{t-1}, \beta^{t}, \gamma^{t}\right) \geq 0 \geq \pi_{1}\left(x_{1}^{t}, \beta^{t+1}, \gamma^{t+1}\right)-\pi_{1}\left(x_{1}^{t-1}, \beta^{t+1}, \gamma^{t+1}\right)
$$

or, equivalently,

$$
-\int_{x_{1}^{t}}^{x_{1}^{t-1}} \frac{\partial \pi_{1}\left(x, \beta^{t}, \gamma^{t}\right)}{\partial x} d x \geq 0 \geq-\int_{x_{1}^{t}}^{x_{1}^{t-1}} \frac{\partial \pi_{1}\left(x, \beta^{t+1}, \gamma^{t+1}\right)}{\partial x} d x
$$

Next note that

$$
\frac{\partial^{2} \pi_{1}}{\partial x \partial \beta}=(1-2 \rho) g^{\prime}(x)<0, \frac{\partial^{2} \pi_{1}}{\partial x \partial \gamma}=-(1-2 \rho) g^{\prime}(1-x)>0 .
$$

Since $\beta^{t+1}>\beta^{t}$ and $\gamma^{t+1}<\gamma^{t}$ from Lemma 9, it follows from (29) that

$$
\frac{\partial \pi_{1}\left(x, \beta^{t}, \gamma^{t}\right)}{\partial x}>\frac{\partial \pi_{1}\left(x, \beta^{t+1}, \gamma^{t+1}\right)}{\partial x}
$$

\footnotetext{
${ }^{21}$ If $\phi_{1}^{t+1} \leq \psi_{1}^{t}$ then case (i-b) disappears.
} 
and therefore

$$
\int_{x_{1}^{t}}^{x_{1}^{t-1}} \frac{\partial \pi_{1}\left(x, \beta^{t}, \gamma^{t}\right)}{\partial x} d x>\int_{x_{1}^{t}}^{x_{1}^{t-1}} \frac{\partial \pi_{1}\left(x, \beta^{t+1}, \gamma^{t+1}\right)}{\partial x} d x
$$

which contradicts (28). This shows our supposition of $x_{1}^{t-1} \leq \psi_{1}^{t+1}$ to be false and thus $\psi_{1}^{t+1}<x_{1}^{t-1}$. This implies

$$
\left(\psi_{1}^{t+1}=\right) \max \left\{\psi_{1}^{t+1}, x_{1}^{t}\right\} \leq \max \left\{\psi_{1}^{t}, x_{1}^{t-1}\right\}\left(=x_{1}^{t-1}\right) .
$$

This proves (25) for when $x_{1}^{t} \in\left[\psi_{1}^{t}, \phi_{1}^{t+1}\right)$.

ii) Suppose $x_{1}^{t-1}<\phi_{1}^{t}$. Hence, $x_{1}^{t-1}<\phi_{1}^{t}<\psi_{1}^{t}$. This implies $x_{1}^{t-1} \leq x_{1}^{t}$. From Lemma $9, \phi_{1}^{t+1}<\phi_{1}^{t}$. Partition the possible values of $x_{1}^{t}$ into $^{22}$

$$
\left[x_{1}^{t-1}, \phi_{1}^{t+1}\right) \cup\left[\phi_{1}^{t+1}, \phi_{1}^{t}\right) \cup\left[\phi_{1}^{t}, \psi_{1}^{t}\right] .
$$

ii-a) Suppose $x_{1}^{t} \in\left[\phi_{1}^{t}, \psi_{1}^{t}\right]$. Since $\phi_{1}^{t+1}<\phi_{1}^{t}$ then $\phi_{1}^{t} \leq x_{1}^{t}$ implies $\phi_{1}^{t+1}<x_{1}^{t}$. It follows that $\psi_{1}^{t+1}<\phi_{1}^{t+1}$. We then have $\psi_{1}^{t+1}<\phi_{1}^{t+1}<\phi_{1}^{t} \leq x_{1}^{t} \leq \psi_{1}^{t}$. Hence, $\max \left\{\psi_{1}^{t+1}, x_{1}^{t}\right\} \leq \max \left\{\psi_{1}^{t}, x_{1}^{t-1}\right\}$.

ii-b) Suppose $x_{1}^{t} \in\left[\phi_{1}^{t+1}, \phi_{1}^{t}\right)$. As then $\psi_{1}^{t+1} \leq \phi_{1}^{t+1} \leq x_{1}^{t}<\phi_{1}^{t}<\psi_{1}^{t}$, it follows that $\max \left\{\psi_{1}^{t+1}, x_{1}^{t}\right\} \leq \max \left\{\psi_{1}^{t}, x_{1}^{t-1}\right\}$.

ii-c) Suppose $x_{1}^{t} \in\left[x_{1}^{t-1}, \phi_{1}^{t+1}\right)$. We then have $x_{1}^{t}<\phi_{1}^{t+1}<\psi_{1}^{t+1}$. We want to prove $\max \left\{\psi_{1}^{t+1}, x_{1}^{t}\right\} \leq \max \left\{\psi_{1}^{t}, x_{1}^{t-1}\right\}$ where, in this case, the lhs is $\psi_{1}^{t+1}$ and the rhs is $\psi_{1}^{t}$. It is then sufficient to show $\psi_{1}^{t+1} \leq \psi_{1}^{t}$. Since $x_{1}^{t}<\psi_{1}^{t}$ and $x_{1}^{t}<\phi_{1}^{t+1}<\psi_{1}^{t+1}$, the strict concavity of the profit function implies that if

$$
\pi_{1}\left(\psi_{1}^{t+1}, \beta^{t+1}, \gamma^{t+1}\right)>\pi_{1}\left(\psi_{1}^{t}, \beta^{t+1}, \gamma^{t+1}\right)
$$

then $\psi_{1}^{t+1}<\psi_{1}^{t}$. First note that

$$
\pi_{1}\left(x_{1}^{t}, \beta^{t}, \gamma^{t}\right) \geq \pi_{1}\left(x_{1}^{t-1}, \beta^{t}, \gamma^{t}\right)=\pi_{1}\left(\psi_{1}^{t}, \beta^{t}, \gamma^{t}\right)
$$

where the last is an equality because $\psi_{1}^{t} \in(0,1) \cdot{ }^{23}$ Next note that $x_{1}^{t}<\psi_{1}^{t}$. Observe that

$$
\begin{gathered}
\pi_{1}\left(x_{1}^{t}, \beta^{t+1}, \gamma^{t+1}\right)-\pi_{1}\left(\psi_{1}^{t}, \beta^{t+1}, \gamma^{t+1}\right) \\
>\pi_{1}\left(x_{1}^{t}, \beta^{t}, \gamma^{t}\right)-\pi_{1}\left(\psi_{1}^{t}, \beta^{t}, \gamma^{t}\right) \Leftrightarrow \\
-\int_{x_{1}^{t}}^{\psi_{1}^{t}} \frac{\partial \pi_{1}\left(x, \beta^{t+1}, \gamma^{t+1}\right)}{\partial x} d x>-\int_{x_{1}^{t}}^{\psi_{1}^{t}} \frac{\partial \pi_{1}\left(x, \beta^{t}, \gamma^{t}\right)}{\partial x} d x \Leftrightarrow
\end{gathered}
$$

\footnotetext{
${ }^{22}$ If $\phi_{1}^{t+1} \leq x_{1}^{t-1}$ then case (ii-a) disappears.

${ }^{23}$ If $\psi_{1}^{t}=1$ then $\psi_{1}^{t+1} \leq \psi_{1}^{t}$ and we're done.
} 


$$
\int_{x_{1}^{t}}^{\psi_{1}^{t}}\left[\frac{\partial \pi_{1}\left(x, \beta^{t}, \gamma^{t}\right)}{\partial x}-\frac{\partial \pi_{1}\left(x, \beta^{t+1}, \gamma^{t+1}\right)}{\partial x}\right] d x>0 .
$$

This last inequality is true because the integrand is positive for all $x$ since: i) $\beta^{t+1}>\beta^{t}$ and $\partial^{2} \pi_{1} / \partial x \partial \beta<0$; and ii) $\gamma^{t+1}<\gamma^{t}$ and $\partial^{2} \pi_{1} / \partial x \partial \gamma>0$. It follows from (34) being true and that the rhs is non-negative that:

$$
\pi_{1}\left(x_{1}^{t}, \beta^{t+1}, \gamma^{t+1}\right)>\pi_{1}\left(\psi_{1}^{t}, \beta^{t+1}, \gamma^{t+1}\right) .
$$

Next note that ${ }^{24}$

$$
\pi_{1}\left(x_{1}^{t}, \beta^{t+1}, \gamma^{t+1}\right) \leq \pi_{1}\left(\psi_{1}^{t+1}, \beta^{t+1}, \gamma^{t+1}\right)
$$

It follows from (35)-(36) that (32) is true. We conclude $\psi_{1}^{t+1} \leq \psi_{1}^{t}$ and thus $\max \left\{\psi_{1}^{t+1}, x_{1}^{t}\right\} \leq \max \left\{\psi_{1}^{t}, x_{1}^{t-1}\right\}$.

We have shown $\max \left\{\psi_{1}^{t+1}, x_{1}^{t}\right\} \leq \max \left\{\psi_{1}^{t}, x_{1}^{t-1}\right\}$. By an analogous argument, one can show $\min \left\{\psi_{2}^{t}, x_{2}^{t-1}\right\} \leq \min \left\{\psi_{2}^{t+1}, x_{2}^{t}\right\}$. It immediately follows that if $\max \left\{\psi_{1}^{t}, x_{1}^{t-1}\right\}<$ $\min \left\{\psi_{2}^{t}, x_{2}^{t-1}\right\}$ then $\max \left\{\psi_{1}^{t+1}, x_{1}^{t}\right\}<\min \left\{\psi_{2}^{t+1}, x_{2}^{t}\right\}$. The proof of Lemma 10 is completed by induction.

Using Lemmas 9-10, we can complete the proof of Theorem 4 by induction. With Lemma 10, we can conclude from Lemma 9 that if $\max \left\{\psi_{1}^{t}, x_{1}^{t-1}\right\}<\min \left\{\psi_{2}^{t}, x_{2}^{t-1}\right\}$ then

$$
\left(\beta^{\tau+1}, \gamma^{\tau+1}\right)=\left(\beta^{\tau}+\rho\left(\alpha-\beta^{\tau}\right),(1-\rho) \gamma^{\tau}\right) \forall \tau \geq t
$$

We claim that this implies $\beta^{\tau}=(1-\rho)^{\tau-t} \beta^{t}+\left[1-(1-\rho)^{\tau-t}\right] \alpha$ and $\gamma^{\tau}=(1-\rho)^{\tau-t} \gamma^{t}$. Suppose it is true for $\tau^{\prime}$. It follows from Lemma 9 that

$$
\begin{aligned}
\beta^{\tau^{\prime}+1}= & \beta^{\tau^{\prime}}+\rho\left(\alpha-\beta^{\tau^{\prime}}\right) \\
= & \left\{(1-\rho)^{\tau^{\prime}-t} \beta^{t}+\left[1-(1-\rho)^{\tau^{\prime}-t}\right] \alpha\right\} \\
& +\rho\left\{\alpha-(1-\rho)^{\tau^{\prime}-t} \beta^{t}-\left[1-(1-\rho)^{\tau^{\prime}-t}\right] \alpha\right\} \\
= & (1-\rho)^{\tau^{\prime}+1-t} \beta^{t}+\left[1-(1-\rho)^{\tau^{\prime}+1-t}\right] \alpha ;
\end{aligned}
$$

which establishes the dynamic on $\beta^{\tau}$. Now suppose $\gamma^{\tau}=(1-\rho)^{\tau-t} \gamma^{t}$ :

$$
\gamma^{\tau^{\prime}+1}=(1-\rho) \gamma^{\tau^{\prime}}=(1-\rho)\left[(1-\rho)^{\tau-t} \gamma^{t}\right]=(1-\rho) \gamma^{\tau^{\prime}+1}
$$

which establishes the dynamic on $\gamma^{\tau}$.

Proof of Theorem 5: To prove this theorem, it needs to be shown that for finite $T>t,\left(x_{1}^{T}, x_{2}^{T}, \beta^{T+1}, \gamma^{T+1}\right) \in \Omega_{1}$ with positive probability and $\left(x_{1}^{T}, x_{2}^{T}, \beta^{T+1}, \gamma^{T+1}\right) \in$

\footnotetext{
${ }^{24}$ If $\psi_{1}^{t+1} \in(0,1)$ then this is an equality and if $\psi_{1}^{t+1}=1$ then it is, generically, a strict inequality.
} 
$\Omega_{2}$ with positive probability. It is shown in the proof of Theorem 6 that, in finite time with positive probability, if $x_{1}^{t-1}<x_{2}^{t-1}$ then the state lies in $\Omega_{1}$ and if $x_{2}^{t-1}<x_{1}^{t-1}$ then the state lies in $\Omega_{2}$. It then just needs to be shown that if $x_{1}^{t-1}<x_{2}^{t-1}$ then the state can end up in $\Omega_{2}$ and if $x_{2}^{t-1}<x_{1}^{t-1}$ then the state can end up in $\Omega_{1}$.

By assumption, $\min \left\{\psi_{2}^{t}, x_{2}^{t-1}\right\}<\max \left\{\psi_{1}^{t}, x_{1}^{t-1}\right\}$. Choose $\varepsilon>0$ such that: 1) if $y_{2}^{t} \in\left(\min \left\{\psi_{2}^{t}, x_{2}^{t-1}\right\}, \min \left\{\psi_{2}^{t}, x_{2}^{t-1}\right\}+\varepsilon\right)$ then $\left.x_{2}^{t}=y_{2}^{t} ; 2\right)$ if $y_{1}^{t} \in\left(\max \left\{\psi_{1}^{t}, x_{1}^{t-1}\right\}-\right.$ $\left.\varepsilon, \max \left\{\psi_{1}^{t}, x_{1}^{t-1}\right\}\right)$ then $x_{1}^{t}=y_{1}^{t}$; and 3) $y_{2}^{t}<y_{1}^{t}$. It follows that, with positive probability, $x_{2}^{t}<x_{1}^{t}$ and, therefore, in finite time with positive probability the state lies in $\Omega_{2}$. A similar argument can be used to show that if $x_{2}^{t-1}<x_{1}^{t-1}$ then the state is in $\Omega_{1}$ in finite time with positive probability.

Proof of Theorem 6: If the state is in $\Omega_{1} \cup \Omega_{2}$ then we know by Theorem 4 that $\lim _{t \rightarrow \infty}\left(\beta^{t}, \gamma^{t}\right) \in\{(\alpha, 0),(0,1-\alpha)\}$. Let us then consider when the state lies in $\bar{\Omega} \equiv[0,1]-\left(\Omega_{1} \cup \Omega_{2}\right)-\left\{\left(x_{1}, x_{2}\right): x_{1}=x_{2}\right\}$. The method of proof is to show $\exists$ finite $T^{*}$ and $\varepsilon^{*} \in(0,1)$ such that for all $\left(x_{1}^{0}, x_{2}^{0}, \beta^{1}, \gamma^{1}\right) \in \bar{\Omega},\left(x_{1}^{T^{*}-1}, x_{2}^{T^{*}-1}, \beta^{T^{*}}, \gamma^{T^{*}}\right) \in \Omega_{1} \cup \Omega_{2}$ with probability exceeding $\varepsilon^{*}$. Therefore, the probability that the state is in $\Omega_{1} \cup \Omega_{2}$ in period $k T^{*}$ exceeds $1-\left(1-\varepsilon^{*}\right)^{k}$ and this goes to one as $k T^{*} \rightarrow \infty$.

Assume wlog that $x_{1}^{0}<x_{2}^{0}$. Recall the following definitions: $\underline{\phi}=\phi(\alpha, 0)$ and $\bar{\phi}=\phi(0,1-\alpha)$; and that $\phi<\bar{\phi}$. Given $x_{1}^{0}<x_{2}^{0}$, if there are no new ideas over periods $1, \ldots, \tau$ then $x_{1}^{t-1}<x_{2}^{t}$ and $x_{1}^{t}<x_{2}^{t-1} \forall t \in\{1, \ldots, \tau\}$ from which it follows that

$$
(\underline{\phi}<) \phi_{1}^{\tau}<\phi_{1}^{\tau-1}<\cdots<\phi_{1}^{1} \text { and } \phi_{2}^{1}<\cdots<\phi_{2}^{\tau-1}<\phi_{2}^{\tau}(<\bar{\phi})
$$

and $\lim _{\tau \rightarrow \infty} \phi_{1}^{\tau}=\underline{\phi}$ and $\lim _{\tau \rightarrow \infty} \phi_{2}^{\tau}=\bar{\phi}$. Since $\underline{\phi}<\bar{\phi}$, it follows that $\exists$ finite $T^{\prime}$ (which is the same $\left.\forall\left(\beta^{0}, \gamma^{0}\right)\right)$ such that if there are no new ideas over periods $1, \ldots, T^{\prime}$ then $\underline{\phi}<\phi_{1}^{T^{\prime}}<\phi_{2}^{T^{\prime}}<\bar{\phi}$. This event occurs with probability $(1-\omega)^{2 T^{\prime}}>0 .{ }^{25}$ Next note that $\left(x_{1}^{0}=\right) x_{1}^{T^{\prime}-1}<x_{2}^{T^{\prime}-1}\left(=x_{2}^{0}\right)$.

There are four cases to consider: i) $\phi_{1}^{T^{\prime}} \leq x_{1}^{T^{\prime}-1}, x_{2}^{T^{\prime}-1} \leq \phi_{2}^{T^{\prime}}$; ii) $x_{1}^{T^{\prime}-1}<\phi_{1}^{T^{\prime}}, \phi_{2}^{T^{\prime}}<$ $x_{2}^{T^{\prime}-1}$; iii) $x_{1}^{T^{\prime}-1}<\phi_{1}^{T^{\prime}}, x_{2}^{T^{\prime}-1} \leq \phi_{2}^{T^{\prime}} ;$ and iv) $\phi_{1}^{T^{\prime \prime}} \leq x_{1}^{T^{\prime}}, \phi_{2}^{T^{\prime}}<x_{2}^{T^{\prime}}$.

i) Suppose $\phi_{1}^{T^{\prime}} \leq x_{1}^{T^{\prime}-1}(<) x_{2}^{T^{\prime}-1} \leq \phi_{2}^{T^{\prime}}$. The state lies in $\Omega_{1}$ and we're done.

ii) Suppose $x_{1}^{T^{\prime}-1}<\phi_{1}^{T^{\prime}}<\phi_{2}^{T^{\prime}}<x_{2}^{T^{\prime}-1}$. Choose $\varepsilon \in\left(0, \min \left\{\phi_{1}^{T^{\prime}}-\underline{\phi}, \bar{\phi}-\phi_{2}^{T^{\prime}}\right\}\right)$. There are four sub-cases to consider: a) $x_{1}^{T^{\prime}-1}<\phi_{1}^{T^{\prime}}-\varepsilon, \phi_{2}^{T^{\prime}}+\varepsilon<x_{2}^{T^{\prime}-1}$; b) $x_{1}^{T^{\prime}-1} \in\left[\phi_{1}^{T^{\prime}}-\varepsilon, \phi_{1}^{T^{\prime}}\right), \phi_{2}^{T^{\prime}}+\varepsilon<x_{2}^{T^{\prime}-1} ;$ c) $x_{1}^{T^{\prime}-1}<\phi_{1}^{T^{\prime}}-\varepsilon, x_{2}^{T^{\prime}-1} \in\left(\phi_{2}^{T^{\prime}}, \phi_{2}^{T^{\prime}}+\varepsilon\right] ;$ and d) $x_{1}^{T^{\prime}-1} \in\left[\phi_{1}^{T^{\prime}}-\varepsilon, \phi_{1}^{T^{\prime}}\right), x_{2}^{T^{\prime}-1} \in\left(\phi_{2}^{T^{\prime}}, \phi_{2}^{T^{\prime}}+\varepsilon\right]$.

ii-a) Suppose $x_{1}^{T^{\prime}-1}<\phi_{1}^{T^{\prime}}-\varepsilon, \phi_{2}^{T^{\prime}}+\varepsilon<x_{2}^{T^{\prime}-1}$. Since $x_{1}^{T^{\prime}-1}$ is bounded below $\phi_{1}^{T^{\prime}}$, it follows that $\psi_{1}^{T^{\prime}}$ is bounded above $\phi_{1}^{T^{\prime}}$. Since $\phi_{1}^{T^{\prime}}$ is bounded

${ }^{25}$ If $\omega=1$ then proofs could be adapted to assume that no acceptable ideas are received for $T^{\prime}$ periods. 
below $\phi_{2}^{T^{\prime}}$ then $\min \left\{\psi_{1}^{T^{\prime}}, .5\left(\phi_{1}^{T^{\prime}}+\phi_{2}^{T^{\prime}}\right)\right\}$ is bounded above $\phi_{1}^{T^{\prime}}$. With probability bounded above zero, store 1 gets a new idea in period $T^{\prime}$ for which $\phi_{1}^{T^{\prime}}<y_{1}^{T^{\prime}}<\min \left\{\psi_{1}^{T^{\prime}}, .5\left(\phi_{1}^{T^{\prime}}+\phi_{2}^{T^{\prime}}\right)\right\}$ and hence $\phi_{1}^{T^{\prime}}<x_{1}^{T^{\prime}}<$ $\min \left\{\psi_{1}^{T^{\prime}}, .5\left(\phi_{1}^{T^{\prime}}+\phi_{2}^{T^{\prime}}\right)\right\}$. By the same argument, with probability bounded above zero, $\max \left\{\psi_{2}^{T^{\prime}}, .5\left(\phi_{1}^{T^{\prime}}+\phi_{2}^{T^{\prime}}\right)\right\}<x_{2}^{T^{\prime}}<\phi_{2}^{T^{\prime}}$. We then have $\phi_{1}^{T^{\prime}}<$ $x_{1}^{T^{\prime}}<x_{2}^{T^{\prime}}<\phi_{2}^{T^{\prime}}$. Finally, since $x_{1}^{T^{\prime}-1}<x_{2}^{T^{\prime}}$ and $x_{1}^{T^{\prime}-1}<x_{2}^{T^{\prime}}$ then $\phi_{1}^{T^{\prime}+1}<\phi_{1}^{T^{\prime}}$ and $\phi_{2}^{T^{\prime}}<\phi_{2}^{T^{\prime}+1}$. We conclude: $\phi_{1}^{T^{\prime}+1}<x_{1}^{T^{\prime}}<x_{2}^{T^{\prime}}<\phi_{2}^{T^{\prime}+1}$; in which case the state is in $\Omega_{1}$ in finite time with positive probability.

ii-b) Suppose $x_{1}^{T^{\prime}-1} \in\left[\phi_{1}^{T^{\prime}}-\varepsilon, \phi_{1}^{T^{\prime}}\right), \phi_{2}^{T^{\prime}}+\varepsilon<x_{2}^{T^{\prime}-1}$. With probability bounded above zero, firm 2 gets a new idea for which $y_{2}^{T^{\prime}} \in\left(\max \left\{\psi_{2}^{T^{\prime}}, \phi_{1}^{T^{\prime}}\right\}, \phi_{2}^{T^{\prime}}\right)$ so that $x_{2}^{T^{\prime}} \in\left(x_{1}^{T^{\prime}}, \phi_{2}^{T^{\prime}}\right) \cdot \exists$ finite $t^{0}$ such that, if there are no new ideas for firm 1 over periods $T^{\prime}, \ldots, T^{\prime}+t^{0}$, then $\phi_{1}^{T^{\prime}+t^{0}}<x_{1}^{T^{\prime}+t^{0}-1}\left(=x_{1}^{T^{\prime}-1}\right)$. (Note that $t^{0}$ is finite because $\phi_{1}^{T^{\prime}}-\varepsilon \leq x_{1}^{T^{\prime}-1}$ and thus $x_{1}^{T^{\prime}-1}$ is bounded above $\phi)$. If there are also no new ideas for store 2 over $T^{\prime}+1, \ldots, T^{\prime}+t^{0}$ then $\left(\bar{x}_{2}^{T^{\prime}}=\right) x_{2}^{T^{\prime}+t^{0}-1}<\phi_{2}^{T^{\prime}}<\phi_{2}^{T^{\prime}+t^{0}}$. We then have

$$
\phi_{1}^{T^{\prime}+t^{0}}<x_{1}^{T^{\prime}+t^{0}-1}<x_{2}^{T^{\prime}+t^{0}-1}<\phi_{2}^{T^{\prime}+t^{0}} .
$$

Thus, starting from period 1 , the state is in $\Omega_{1}$ in period $T^{\prime}+t^{0}$ with positive probability.

ii-c) Suppose $x_{1}^{T^{\prime}-1}<\phi_{1}^{T^{\prime}}-\varepsilon, x_{2}^{T^{\prime}-1} \in\left[\phi_{2}^{T^{\prime}}, \phi_{2}^{T^{\prime}}+\varepsilon\right]$. The proof is analogous to that in (ii-b). One can show $\exists$ finite $t^{00}$ such that with probability bounded above zero,

$$
\phi_{1}^{T^{\prime}+t^{00}}<x_{1}^{T^{\prime}+t^{00}-1}<x_{2}^{T^{\prime}+t^{00}-1}<\phi_{2}^{T^{\prime}+t^{00}} .
$$

Thus, starting from period 1 , the state is in $\Omega_{1}$ in period $T^{\prime}+t^{00}$ with positive probability.

ii-d) Suppose $x_{1}^{T^{\prime}-1} \in\left[\phi_{1}^{T^{\prime}}-\varepsilon, \phi_{1}^{T^{\prime}}\right), x_{2}^{T^{\prime}-1} \in\left(\phi_{2}^{T^{\prime}}, \phi_{2}^{T^{\prime}}+\varepsilon\right]$. By the definition of $t^{0}$ and $t^{00}$, we know that if there are no new ideas for both stores over periods $T^{\prime}, \ldots, T^{\prime}+t^{\prime}$, where $t^{\prime} \equiv \max \left\{t^{0}, t^{00}\right\}$, then

$$
\phi_{1}^{T^{\prime}+t^{\prime}}<x_{1}^{T^{\prime}+t^{\prime}-1}<x_{2}^{T^{\prime}+t^{\prime}-1}<\phi_{2}^{T^{\prime}+t^{\prime}} .
$$

iii) Suppose $x_{1}^{T^{\prime}-1}<\phi_{1}^{T^{\prime}}, x_{2}^{T^{\prime}-1} \leq \phi_{2}^{T^{\prime}}$. We know that $x_{1}^{T^{\prime}-1}<x_{2}^{T^{\prime}-1}$ and $\phi_{1}^{T^{\prime}}<\phi_{2}^{T^{\prime}}$. There are two sub-cases to consider: a) $x_{1}^{T^{\prime}-1}<\phi_{1}^{T^{\prime}} \leq x_{2}^{T^{\prime}-1} \leq \phi_{2}^{T^{\prime}}$; and b) $x_{1}^{T^{\prime}-1}<x_{2}^{T^{\prime}-1}<\phi_{1}^{T^{\prime}}<\phi_{2}^{T^{\prime}}$. 
iii-a) Suppose $x_{1}^{T^{\prime}-1}<\phi_{1}^{T^{\prime}} \leq x_{2}^{T^{\prime}-1} \leq \phi_{2}^{T^{\prime}}$. If there are no new ideas in $T^{\prime}$ then either: i) $\phi_{1}^{T^{\prime}+1}<x_{1}^{T^{\prime}}<x_{2}^{T^{\prime}} \leq \phi_{2}^{T^{\prime}+1}$; or ii) $x_{1}^{T^{\prime}} \leq \phi_{1}^{T^{\prime}+1}<x_{2}^{T^{\prime}} \leq \phi_{2}^{T^{\prime}+1}$. If (i) then we're done. If (ii) then use the arguments in (ii-b)-(ii-c) to show that $\exists$ finite $t^{0}$ such that with probability bounded above zero, $\phi_{1}^{T^{\prime}+t^{0}}<x_{1}^{T^{\prime}+t^{0}-1}$. Keeping firm 2's practices fixed during that time, we end up with:

$$
\phi_{1}^{T^{\prime}+t^{0}}<x_{1}^{T^{\prime}+t^{0}-1}<x_{2}^{T^{\prime}+t^{0}-1}<\phi_{2}^{T^{\prime}+t^{0}} .
$$

iii-b) Suppose $x_{1}^{T^{\prime}-1}<x_{2}^{T^{\prime}-1}<\phi_{1}^{T^{\prime}}<\phi_{2}^{T^{\prime}}$. The probability that $y_{2}^{T^{\prime}} \in\left(.5\left(\phi_{1}^{T^{\prime}}+\phi_{2}^{T^{\prime}}\right), \phi_{2}^{T^{\prime}}\right)$ is bounded above zero. With positive probability, firm 1 does not get an idea. Suppose both of those events happen. We either have: i) $\phi_{1}^{T^{\prime}+1}<x_{1}^{T^{\prime}}<x_{2}^{T^{\prime}}<\phi_{2}^{T^{\prime}+1}$; or ii) $x_{1}^{T^{\prime}} \leq \phi_{1}^{T^{\prime}+1}<x_{2}^{T^{\prime}}<\phi_{2}^{T^{\prime}+1}$. If (i) then we're done. If (ii) then use the arguments in (ii-b)-(ii-c) to show that $\exists$ finite $t^{0}$ such that with probability bounded above zero:

$$
\phi_{1}^{T^{\prime}+t^{0}}<x_{1}^{T^{\prime}+t^{0}-1}<x_{1}^{T^{\prime}+t^{0}-1}<\phi_{2}^{T^{\prime}+t^{0}} .
$$

iv) Suppose $\phi_{1}^{T^{\prime}} \leq x_{1}^{T^{\prime}}, \phi_{2}^{T^{\prime}}<x_{2}^{T^{\prime}}$. The proof is analogous to (iii).

By the preceding steps, we conclude that there is finite time (bounded from above) and positive probability (bounded above zero) such that the state is in $\Omega_{1}$. All this is shown for $x_{1}^{0}<x_{2}^{0}$. If instead $x_{2}^{0}<x_{1}^{0}$ then an analogous set of arguments apply to show that there is finite time (bounded from above) and positive probability (bounded above zero) such that the state is in $\Omega_{2}$. It follows that, almost surely, $\left(x_{1}^{t-1}, x_{2}^{t-1}, \beta^{t}, \gamma^{t}\right) \in \Omega_{1} \cup \Omega_{2}$ as $t \rightarrow \infty$.

Proof of Theorem 7: When the state is such that firms have identical qualities, the model with quality behaves identically to the model without quality (as the models are identical). The idea is to suppose that qualities are identical for a sufficiently long period of time (which we can by A4-A5) so that, with probability bounded above zero, the distance in firms' ideal locations, $\left|\phi_{1}^{t}-\phi_{2}^{t}\right|$, is sufficiently close to $|\bar{\phi}-\underline{\phi}|$. Then, with positive probability, one can get each firm's location sufficiently close to its ideal location so that $\left|\max \left\{\psi_{i}^{t}, x_{i}^{t-1}\right\}-\min \left\{\psi_{j}^{t}, x_{j}^{t-1}\right\}\right|$ is close to $\left|\phi_{1}^{t}-\phi_{2}^{t}\right|$ (for some $i$ ) and, therefore, $\left|\max \left\{\psi_{i}^{t}, x_{i}^{t-1}\right\}-\min \left\{\psi_{j}^{t}, x_{j}^{t-1}\right\}\right|$ is close to $|\bar{\phi}-\underline{\phi}|$. Since $|\bar{\phi}-\underline{\phi}|>\bar{z}$, we can do this so that $\left|\max \left\{\psi_{i}^{t}, x_{i}^{t-1}\right\}-\min \left\{\psi_{j}^{t}, x_{j}^{t-1}\right\}\right|>\bar{z}$. At that point, the distance in firms' locations exceeds $\bar{z}$ so that the quality differential does not affect the consumer dynamics. From this point onward, the dynamics are exactly as specified in the model without quality where we showed that it converges to one firm having all type 0 consumers and the other firm having all type 1 consumers.

From the proof of Theorem 5 , we showed $\exists$ finite $T^{*}$ and $\varepsilon^{*} \in(0,1)$ such that for all $\left(x_{1}^{0}, x_{2}^{0}, \beta^{1}, \gamma^{1}\right) \in \bar{\Omega},\left(x_{1}^{T^{*}-1}, x_{2}^{T^{*}-1}, \beta^{T^{*}}, \gamma^{T^{*}}\right) \in \Omega_{1} \cup \Omega_{2}$ with probability exceeding $\varepsilon^{*}$. Though derived for the model without quality, it applies to the model with qualities 
as long as stores have the same quality. Thus, suppose the quality differential is the same over periods $t-1, \ldots, t+T^{*}$ :

$$
z_{1}^{t-1}-z_{2}^{t-1}=\cdots=z_{1}^{t+T^{*}}-z_{2}^{t+T^{*}}=0 .
$$

Let $\kappa(\tau)$ be a lower bound on the probability that firms have identical qualities over $\tau$ periods. We know that, for each $\tau, \exists \kappa(\tau)$ bounded above zero by A4-A5. The above event occurs with probability of at least $\kappa\left(T^{*}+1\right)$. We can then conclude that from any arbitrary state in period $t$,

$$
\left(x_{1}^{t+T^{*}-1}, x_{2}^{t+T^{*}-1}, \beta^{t+T^{*}}, \gamma^{t+T^{*}}\right) \in \Omega_{1} \cup \Omega_{2}
$$

and $z_{1}^{t+T^{*}-1}-z_{2}^{t+T^{*}-1}=0$ with probability exceeding $\kappa\left(T^{*}+1\right) \varepsilon^{*}$.

If qualities remain the same for the remainder of the horizon then, by Theorem 6 , it follows that

$$
\lim _{\tau \rightarrow \infty}\left(\phi_{1}^{t+T^{*}+\tau}, \phi_{2}^{t+T^{*}+\tau}\right) \in\{(\underline{\phi}, \bar{\phi}),(\bar{\phi}, \underline{\phi})\}
$$

Wlog, suppose $\lim _{\tau \rightarrow \infty}\left(\phi_{1}^{t+T^{*}+\tau}, \phi_{2}^{t+T^{*}+\tau}\right)=(\underline{\phi}, \bar{\phi})$. (The entire proof can be redone for when the other case occurs.) Define $\delta \equiv \bar{\phi}-\underline{\phi}-\bar{z}$. By assumption, $\delta>0$. There then exists finite $\bar{T}$ such that

$$
\phi_{1}^{t+T^{*}+\tau}-\underline{\phi} \in\left(0, \frac{\delta}{4}\right) \text { and } \bar{\phi}-\phi_{2}^{t+T^{*}+\tau} \in\left(0, \frac{\delta}{4}\right) \forall \tau \geq \bar{T}
$$

as long as qualities remain identical. Qualities remain identical over periods $t, \ldots, t+$ $T^{*}+\bar{T}$ with probability of at least $\kappa\left(T^{\prime}\right)$ where $T^{\prime} \equiv T^{*}+\bar{T}$. Therefore, with probability of at least $\kappa\left(T^{\prime}+1\right) \varepsilon^{*}$, we have:

$$
\phi_{1}^{t+T^{\prime}}-\underline{\phi} \in\left(0, \frac{\delta}{4}\right) \text { and } \bar{\phi}-\phi_{2}^{t+T^{\prime}} \in\left(0, \frac{\delta}{4}\right)
$$

Choose $\eta \in\left(0, \frac{\delta}{4}\right)$. There are four cases to consider:

\begin{tabular}{|l|l|}
\hline i) $\max \left\{\psi_{1}^{t+T^{\prime}}, x_{1}^{t+T^{\prime}-1}\right\}<\phi_{1}^{t+T^{\prime}}+\eta$ & ii) $\max \left\{\psi_{1}^{t+T^{\prime}}, x_{1}^{t+T^{\prime}-1}\right\}>\phi_{1}^{t+T^{\prime}}+\eta$ \\
and $\min \left\{\psi_{2}^{t+T^{\prime}}, x_{2}^{t+T^{\prime}-1}\right\}>\phi_{2}^{t+T^{\prime}}-\eta$ & and $\min \left\{\psi_{2}^{t+T^{\prime}}, x_{2}^{t+T^{\prime}-1}\right\}>\phi_{2}^{t+T^{\prime}}-\eta$ \\
\hline iii) $\max \left\{\psi_{1}^{t+T^{\prime}}, x_{1}^{t+T^{\prime}-1}\right\}<\phi_{1}^{t+T^{\prime}}+\eta$ & iv) $\max \left\{\psi_{1}^{t+T^{\prime}}, x_{1}^{t+T^{\prime}-1}\right\}>\phi_{1}^{t+T^{\prime}}+\eta$ \\
and $\min \left\{\psi_{2}^{t+T^{\prime}}, x_{2}^{t+T^{\prime}-1}\right\}<\phi_{1}^{t+T^{\prime}}-\eta$ & and $\min \left\{\psi_{2}^{t+T^{\prime}}, x_{2}^{t+T^{\prime}-1}\right\}<\phi_{2}^{t+T^{\prime}}-\eta$ \\
\hline
\end{tabular}

i) Suppose

$$
\max \left\{\psi_{1}^{t+T^{\prime}}, x_{1}^{t+T^{\prime}-1}\right\}<\phi_{1}^{t+T^{\prime}}+\eta \text { and } \min \left\{\psi_{2}^{t+T^{\prime}}, x_{2}^{t+T^{\prime}-1}\right\}>\phi_{2}^{t+T^{\prime}}-\eta .
$$


It follows:

$$
\begin{aligned}
& \min \left\{\psi_{2}^{t+T^{\prime}}, x_{2}^{t+T^{\prime}-1}\right\}-\max \left\{\psi_{1}^{t+T^{\prime}}, x_{1}^{t+T^{\prime}-1}\right\} \\
> & \phi_{2}^{t+T^{\prime}}-\eta-\phi_{1}^{t+T^{\prime}}-\eta>\phi_{2}^{t+T^{\prime}}-\phi_{1}^{t+T^{\prime}}-\frac{\delta}{2} \\
> & \left(\bar{\phi}-\frac{\delta}{4}-\underline{\phi}-\frac{\delta}{4}\right)-\frac{\delta}{2}>\bar{\phi}-\underline{\phi}-\delta=\bar{z} .
\end{aligned}
$$

Since $x_{1}^{t+T^{\prime}} \leq \max \left\{\psi_{1}^{t+T^{\prime}}, x_{1}^{t+T^{\prime}-1}\right\}$ and $\min \left\{\psi_{2}^{t+T^{\prime}}, x_{2}^{t+T^{\prime}-1}\right\} \leq x_{2}^{t+T^{\prime}-1}$, it follows from (39) that $\bar{z}<x_{2}^{t+T^{\prime}-1}-x_{1}^{t+T^{\prime}}$. Since $x_{1}^{t+T^{\prime}-1} \leq \max \left\{\psi_{1}^{t+T^{\prime}}, x_{1}^{t+T^{\prime}-1}\right\}$ and $\min \left\{\psi_{2}^{t+T^{\prime}}, x_{2}^{t+T^{\prime}-1}\right\} \leq x_{2}^{t+T^{\prime}}$, it follows from (39) that $\bar{z}<x_{2}^{t+T^{\prime}}-x_{1}^{t+T^{\prime}-1}$. Hence, regardless of the realizations of $\left(z_{1}^{t+T^{\prime}}, z_{2}^{t+T^{\prime}}\right)$, type 0 (1) customers who are loyal in the current period to firm 1 (2) will remain so and type 0 (1) customers who are loyal in the current period to firm 2 (1) but buy from firm 1 (2) will switch their loyalty to firm 1 (2). The equation of motion is then

$$
\left(\beta^{t+T^{\prime}+1}, \gamma^{t+T^{\prime}+1}\right)=\left(\beta^{t+T^{\prime}}+\rho\left(\alpha-\beta^{t+T^{\prime}}\right),(1-\rho) \gamma^{t+T^{\prime}}\right)
$$

From the proof of Theorem 4, we know that when this is the equation of motion then $\max \left\{\psi_{1}^{t+T^{\prime}+1}, x_{1}^{t+T^{\prime}}\right\} \leq \max \left\{\psi_{1}^{t+T^{\prime}}, x_{1}^{t+T^{\prime}-1}\right\}$ and $\min \left\{\psi_{2}^{t+T^{\prime}}, x_{2}^{t+T^{\prime}-1}\right\} \leq$ $\min \left\{\psi_{2}^{t+T^{\prime}+1}, x_{2}^{t+T^{\prime}}\right\}$. Using the induction argument from the proof of Theorem $6, \lim _{\tau \rightarrow \infty}\left(\beta^{t+T^{\prime}+\tau}, \gamma^{t+T^{\prime}+\tau}\right) \in\{(\alpha, 0),(0,1-\alpha)\}$.

ii) Suppose

$$
\max \left\{\psi_{1}^{t+T^{\prime}}, x_{1}^{t+T^{\prime}-1}\right\}>\phi_{1}^{t+T^{\prime}}+\eta \text { and } \min \left\{\psi_{2}^{t+T^{\prime}}, x_{2}^{t+T^{\prime}-1}\right\}>\phi_{1}^{t+T^{\prime}}-\eta .
$$

Recall that $\max \left\{\psi_{1}^{t+T^{\prime}}, x_{1}^{t+T^{\prime}-1}\right\}<\min \left\{\psi_{2}^{t+T^{\prime}}, x_{2}^{t+T^{\prime}-1}\right\} \cdot \exists \xi>(0, \eta)$ such that if $y_{1}^{t+T^{\prime}} \in\left(\phi_{1}^{t+T^{\prime}}, \phi_{1}^{t+T^{\prime}}+\xi\right)$ then $x_{1}^{t+T^{\prime}}=y_{1}^{t+T^{\prime}}$. Since $\eta$ is bounded above zero then we can choose $\xi$ so that the event $y_{1}^{t+T^{\prime}} \in\left(\phi_{1}^{t+T^{\prime}}, \phi_{1}^{t+T^{\prime}}+\xi\right)$ has probability bounded above zero. Suppose qualities remain identical. Since $x_{1}^{t+T^{\prime}}<x_{2}^{t+T^{\prime}-1}$ and $x_{1}^{t+T^{\prime}}<x_{2}^{t+T^{\prime}-1}$ then $\phi_{1}^{t+T^{\prime}+1}<\phi_{1}^{t+T^{\prime}}$. Since $\phi_{1}^{t+T^{\prime}+1}<$ $x_{1}^{t+T^{\prime}}$ then $\max \left\{\psi_{1}^{t+T^{\prime}+1}, x_{1}^{t+T^{\prime}}\right\}=x_{1}^{t+T^{\prime}}$ from which we conclude

$$
\max \left\{\psi_{1}^{t+T^{\prime}+1}, x_{1}^{t+T^{\prime}}\right\}<\phi_{1}^{t+T^{\prime}+1}+\xi<\phi_{1}^{t+T^{\prime}}+\eta
$$

Finally, suppose firm 2 does not get a new idea, so that $x_{2}^{t+T^{\prime}}=x_{2}^{t+T^{\prime}-1}$. Hence,

$$
\phi_{1}^{t+T^{\prime}}-\eta<\min \left\{\psi_{2}^{t+T^{\prime}}, x_{2}^{t+T^{\prime}-1}\right\} \leq \min \left\{\psi_{2}^{t+T^{\prime}+1}, x_{2}^{t+T^{\prime}}\right\}
$$


We are now in case (i) so we're done.

iii) Suppose $\max \left\{\psi_{1}^{t+T^{\prime}}, x_{1}^{t+T^{\prime}-1}\right\}<\phi_{1}^{t+T^{\prime}}+\eta$ and $\min \left\{\psi_{2}^{t+T^{\prime}}, x_{2}^{t+T^{\prime}-1}\right\}<\phi_{1}^{t+T^{\prime}}-\eta$. The proof is exactly as in case (ii) except that the roles of firms 1 and 2 are reversed.

iv) Suppose $\max \left\{\psi_{1}^{t+T^{\prime}}, x_{1}^{t+T^{\prime}-1}\right\}>\phi_{1}^{t+T^{\prime}}+\eta$ and $\min \left\{\psi_{2}^{t+T^{\prime}}, x_{2}^{t+T^{\prime}-1}\right\}<\phi_{1}^{t+T^{\prime}}-\eta$.

The proof involves using the method deployed for firm 1 in case (ii) on both firms 1 and 2 .

Proof of Theorem 8: We will show that $\exists$ finite $T_{i}$ and $\varepsilon_{i}>0$ such that, for all $s^{t}, s^{t+T_{i}} \in \Gamma_{i}$ with probability of at least $\varepsilon_{i}, i \in\{1,2,3,4\}$. Hence, the probability that the state is in $\Gamma_{i}$ within $k T_{i}$ periods is at least $1-\left(1-\varepsilon_{i}\right)^{k}$. It follows that, almost surely, the state is in $\Gamma_{i}$ in finite time. There are two cases to consider: $\mathrm{i}$ ) $\left|x_{1}^{t-1}-x_{2}^{t-1}\right|>\bar{z}$; and ii) $\left|x_{1}^{t-1}-x_{2}^{t-1}\right|<\bar{z}$.

i) Suppose $\left|x_{1}^{t-1}-x_{2}^{t-1}\right|>\bar{z}$. Since, by assumption, $\bar{z}>\bar{\phi}-\underline{\phi}$ then $\left|x_{1}^{t-1}-x_{2}^{t-1}\right|>$ $\bar{\phi}-\phi$. Given $\Gamma_{1}$ and $\Gamma_{2}$ are identical except for a change in the numbering of firms and $\Gamma_{3}$ and $\Gamma_{4}$ are identical except for a change in the numbering of firms then it is wlog that we assume $x_{1}^{t-1}<x_{2}^{t-1}$. (i-a) shows that the state is in $\Gamma_{1}$ in finite time with positive probability. (i-b) shows it is in $\Gamma_{3}$ and $\Gamma_{4}$. (i-c) shows it is in $\Gamma_{2}$.

i-a) Since $x_{2}^{t-1}-x_{1}^{t-1}>\bar{z} \geq z_{2}^{t-1}-z_{1}^{t-1}$ then type 1 consumers prefer firm 2 and type 0 consumers prefer firm 1 . If the quality differential and firms' locations are unchanged over periods $t-1, \ldots, t+\tau^{\prime}$ (here we are using $\omega<1$ and A5), consumer dynamics are given by

$$
\left(\beta^{t+\tau+1}, \gamma^{t+\tau+1}\right)=\left(\beta^{t+\tau}+\rho\left(\alpha-\beta^{t+\tau}\right),(1-\rho) \gamma^{t+\tau}\right), \tau \in\left\{0,1, \ldots, \tau^{\prime}\right\} .
$$

Note that these dynamics converge to $(\alpha, 0)$. Next note that convergence is slowest for when $\beta^{t}=0$ and $\gamma^{t}=1-\alpha$. However, even for that case, $\exists$ finite $T$ such that $\beta^{t+T}>\frac{\alpha}{2}$ and $\gamma^{t+T}<\frac{1-\alpha}{2}$. Thus, regardless of the initial state, $\exists$ finite $T$ such that with probability of at least $(1-\omega)^{2 T}$, the state in period $t+T$ lies in $\Gamma_{1}$.

i-b) Recall that $\phi_{1}^{t}, \phi_{2}^{t} \in(\underline{\phi}, \bar{\phi})$. Since $x_{2}^{t-1}-x_{1}^{t-1}>\bar{z}>\bar{\phi}-\underline{\phi}$ then $\exists \eta \in$ $\left(0, \frac{\bar{z}-(\bar{\phi}-\underline{\phi})}{2}\right)$ such that $x_{1}^{t-1}<\underline{\phi}-\eta$ and/or $\bar{\phi}+\eta<x_{2}^{t-1}$. We will first show that $0<x_{2}^{t}-x_{1}^{t}<\bar{z}$ with probability bounded above zero and then argue that, in finite time with positive probability bounded above zero, the state is in $\Gamma_{i}$ for $i=3,4$. There are three generic sub-cases to consider: 1) $x_{1}^{t-1}<\underline{\phi}-\eta$ and $x_{2}^{t-1}<\bar{\phi}+\eta$; 2) $x_{1}^{t-1}<\underline{\phi}-\eta$ and $\bar{\phi}+\eta<x_{2}^{t-1}$; and 3) $\underline{\phi}-\eta<x_{1}^{t-1}$ and $\bar{\phi}+\eta<x_{2}^{t-1}$. 
i-b-1) Suppose $x_{1}^{t-1}<\phi-\eta$ and $x_{2}^{t-1}<\bar{\phi}+\eta$. As it follows that $x_{1}^{t-1}<\phi_{1}^{t}$ then $x_{1}^{t}=y_{1}^{t}$ iff $y_{1}^{t} \bar{\epsilon}\left(x_{1}^{t-1}, \psi_{1}^{t}\right)$. Given that $(\phi-\eta, \phi) \subset\left(x_{1}^{t-1}, \psi_{1}^{t}\right)$, if $y_{1}^{t} \in(\underline{\phi}-\eta, \underline{\phi})$ then $x_{1}^{t}=y_{1}^{t}$. This occurs with probability $\omega[F(\underline{\phi})-F(\underline{\phi}-\eta)]$ which is bounded above zero. We need to consider two sub-cases. In sub-case $1^{\prime}, x_{2}^{t-1}<\underline{\phi}$. With probability $\omega\left[F\left(\phi_{2}^{t}\right)-F(\underline{\phi})\right], y_{2}^{t} \in$ $\left(\underline{\phi}, \phi_{2}^{t}\right)$ and therefore $x_{2}^{t}=y_{2}^{t}$. In sub-case $2^{\prime}, \underline{\phi}<x_{2}^{t-1}$ and, with probability $1-\omega, x_{2}^{t}=x_{2}^{t-1}$. In both cases, we have $\underline{\phi}-\eta<x_{1}^{t}<\underline{\phi}<x_{2}^{t}<$ $\bar{\phi}+\eta$. Hence, $x_{2}^{t}-x_{1}^{t}<\bar{\phi}-\phi+2 \eta<\bar{z}$.

i-b-2) Suppose $x_{1}^{t-1}<\underline{\phi}-\eta$ and $\bar{\phi}+\eta<x_{2}^{t-1}$. By the argument in (i-b-1), $\phi-\eta<x_{1}^{t}<\bar{\phi}$. Using this same argument for firm 2, we have $x_{2}^{t} \in(\bar{\phi}, \bar{\phi}+\eta)$. Hence, $\underline{\phi}-\eta<x_{1}^{t}<x_{2}^{t}<\bar{\phi}+\eta$ and thus $0<x_{2}^{t}-x_{1}^{t}<\bar{z}$.

i-b-3) Suppose $\phi-\eta<x_{1}^{t-1}$ and $\bar{\phi}+\eta<x_{2}^{t-1}$. We then just need to reverse the roles in (i-b-1) to get $\underline{\phi}-\eta<x_{1}^{t}<\bar{\phi}<x_{2}^{t}<\bar{\phi}+\eta$ and thus $0<x_{2}^{t}-x_{1}^{t}<\bar{z}$.

We conclude that, with probability bounded above zero, $0<x_{2}^{t}-x_{1}^{t}<\bar{z}$ and therefore with positive probability (by A4), $z_{1}^{t}-z_{2}^{t}>x_{2}^{t}-x_{1}^{t}$ so that all consumer types prefer firm 1 . As long as firms' locations and qualities remain fixed then $\left(\beta^{t+\tau}, \gamma^{t+\tau}\right)$ is converging to $(\alpha, 1-\alpha)$. Thus, in finite time with positive probability, the state lies in $\Gamma_{3}$. With positive probability, $z_{1}^{t}-z_{2}^{t}<x_{1}^{t}-x_{2}^{t}<0$ so that all consumer types prefer firm 2. As long as firms' locations and qualities remain fixed then $\left(\beta^{t+\tau}, \gamma^{t+\tau}\right)$ is converging to $(0,0)$. Thus, in finite time with positive probability, the state lies in $\Gamma_{4}$.

i-c) Continue with the construction in (i-b) in which case $x_{1}^{t}<x_{2}^{t}, x_{2}^{t}-x_{1}^{t}<\bar{z}$, and $\left|x_{1}^{t}-x_{2}^{t}\right|<\left|z_{1}^{t}-z_{2}^{t}\right|$. This implies that both consumer types prefer the same firm. If firms' locations and qualities do not change then a firm's mix of loyal customers is converging to either $(\alpha, 1-\alpha)$ or $(0,0)$. It follows that a firm's mix of customers who visit it each period is converging to either $(1-\rho) \alpha$ of type 0 customers and $(1-\rho)(1-\alpha)$ of type 1 customers or $\rho \alpha$ of type 0 customers and $\rho(1-\alpha)$ of type 1 customers. Therefore, $\left(\phi_{1}^{t+T+\tau}, \phi_{2}^{t+T+\tau}\right)$ is converging to $(\widehat{\phi}, \widehat{\phi})$, where $\widehat{\phi}=$ $\phi(\alpha, 1-\alpha)=\phi(0,0)$, as $\tau \rightarrow \infty$. Hence, $\exists$ finite $T^{\prime}$ such that either: i-c1) $\phi_{1}^{t+T^{\prime}}, \phi_{2}^{t+T^{\prime}}<x_{1}^{t+T^{\prime}-1}<x_{2}^{t+T^{\prime}-1}$; i-c-2) $x_{1}^{t+T^{\prime}-1}<\phi_{1}^{t+T^{\prime}}, \phi_{2}^{t+T^{\prime}}<x_{2}^{t+T^{\prime}-1}$; or i-c-3) $x_{1}^{t+T^{\prime}-1}<x_{2}^{t+T^{\prime}-1}<\phi_{1}^{t+T^{\prime}}, \phi_{2}^{t+T^{\prime}}$. Note that we use the fact that we can make $\phi_{1}^{t+T+\tau}$ and $\phi_{2}^{t+T+\tau}$ as close as we like since they are both converging on the same value. For each of these cases, we want to prove that $\exists$ finite $\tau$ such that $x_{2}^{t+T^{\prime}+\tau}<x_{1}^{t+T^{\prime}+\tau}$ with probability bounded above zero. From that point, we'll argue that the state will get into $\Gamma_{2}$.

i-c-1) Suppose $\phi_{1}^{t+T^{\prime}}, \phi_{2}^{t+T^{\prime}}<x_{1}^{t+T^{\prime}-1}<x_{2}^{t+T^{\prime}-1}$. Assume firm 1 receives no 
new idea and firm 2 receives a new idea with $y_{2}^{t+T^{\prime}} \in\left(\phi_{2}^{t+T^{\prime}}, x_{1}^{t+T^{\prime}-1}\right)$.

We then have, with probability bounded above zero, $x_{2}^{t+T^{\prime}}<x_{1}^{t+T^{\prime}}$.

i-c-2) Suppose $x_{1}^{t+T^{\prime}-1}<\phi_{1}^{t+T^{\prime}}, \phi_{2}^{t+T^{\prime}}<x_{2}^{t+T^{\prime}-1}$. By choosing $T^{\prime}$ so that $\phi_{1}^{t+T^{\prime}}$ and $\phi_{2}^{t+T^{\prime}}$ are sufficiently close, it follows from $x_{1}^{t+T^{\prime}-1}<\phi_{1}^{t+T^{\prime}}, \phi_{2}^{t+T^{\prime}}<$ $x_{2}^{t+T^{\prime}-1}$ that $\psi_{2}^{t+T^{\prime}}<\psi_{1}^{t+T^{\prime}}$. We then have $x_{1}^{t+T^{\prime}-1}<\phi_{1}^{t+T^{\prime}}<\psi_{1}^{t+T^{\prime}}$ and $\psi_{2}^{t+T^{\prime}}<\phi_{2}^{t+T^{\prime}}<x_{2}^{t+T^{\prime}-1}$. Let firm 1 receive a new idea of $y_{1}^{t+T^{\prime}} \in$ $\left(\psi_{2}^{t+T^{\prime}}, \psi_{1}^{t+T^{\prime}}\right)$ and firm 2 receives a new idea of $y_{2}^{t+T^{\prime}} \in\left(\psi_{2}^{t+T^{\prime}}, y_{1}^{t+T^{\prime}}\right)$. Thus, with probability bounded above zero, $x_{2}^{t+T^{\prime}}<x_{1}^{t+T^{\prime}}$.

i-c-3) Suppose $x_{1}^{t+T^{\prime}-1}<x_{2}^{t+T^{\prime}-1}<\phi_{1}^{t+T^{\prime}}, \phi_{2}^{t+T^{\prime}}$. Assume firm 2 receives no new idea and firm 1 receives a new idea $y_{1}^{t+T^{\prime}} \in\left(x_{2}^{t+T^{\prime}-1}, \phi_{1}^{t+T^{\prime}}\right)$. With probability bounded above zero, $x_{2}^{t+T^{\prime}}<x_{1}^{t+T^{\prime}}$.

We have then shown that $\exists$ finite $T^{\prime}$ such that $x_{2}^{t+T^{\prime}}<x_{1}^{t+T^{\prime}}$ with probability bounded above zero. Now suppose that qualities are identical, $z_{1}^{t+T^{\prime}}-z_{2}^{t+T^{\prime}}=0$; which occurs with positive probability by A4. If firms' locations and qualities remain unchanged then, in finite time with probability bounded above zero, the state lies in $\Gamma_{2}$.

In sum, if $\left|x_{1}^{t-1}-x_{2}^{t-1}\right|>\bar{z}$ and $x_{1}^{t-1}<x_{2}^{t-1}$ then $\exists$ finite $T_{i}$ and $\varepsilon_{i}>0$ such that, regardless of the state in period $t$, the state is in $\Gamma_{i}$ in period $t+T_{i}$ with probability of at least $\varepsilon_{i}$.

ii) Suppose $\left|x_{1}^{t-1}-x_{2}^{t-1}\right|<\bar{z}$. With positive probability, $z_{1}^{t}-z_{2}^{t}>\left|x_{1}^{t}-x_{2}^{t}\right|$ so that all consumer types prefer firm 1 . As long as firms' locations and qualities remain fixed then $\left(\beta^{t+\tau}, \gamma^{t+\tau}\right)$ is converging to $(\alpha, 1-\alpha)$. Thus, in finite time with positive probability, the state lies in $\Gamma_{3}$. With positive probability, $z_{2}^{t}-z_{1}^{t}>$ $\left|x_{1}^{t}-x_{2}^{t}\right|$. As long as firms' locations and qualities remain fixed then $\left(\beta^{t+\tau}, \gamma^{t+\tau}\right)$ is converging to $(0,0)$. Thus, in finite time with positive probability, the state lies in $\Gamma_{4}$. Once in either $\Gamma_{3}$ or $\Gamma_{4}$, the argument in (i-c) can be used to get to either $\Gamma_{1}$ or $\Gamma_{2}$ in finite time with positive probability. 


\section{References}

[1] Alós-Ferrer, Carlos, Ana B. Ania, and Klaus Reiner Schenk-Hoppé, "An Evolutionary Model of Bertrand Oligopoly," Games and Economic Behavior, 33 (2000), 1-19.

[2] Bagwell, Kyle, Garey Ramey, and Daniel F. Spulber, "Dynamic Retail Price and Investment Competition," RAND Journal of Economics, 28 (1997), 207-227.

[3] Bergemann, Dirk and Juuso Välimäki, "Market Diffusion with Two-Sided Learning," RAND Journal of Economics, 28 (1997), 773-795.

[4] Budd, Christopher, Christopher Harris, and John Vickers, "A Model of the Evolution of Duopoly: Does the Asymmetry between Firms Tend to Increase or Decrease?," Review of Economic Studies, 60 (1993), 543-573.

[5] Burdett, Kenneth and Melvyn G. Coles, "Steady State Price Distributions in a Noisy Search Equilibrium, Journal of Economic Theory, 72 (1997), 1-32.

[6] Cabral, Luis and Michael Riordan, "The Learning Curve, Market Dominance, and Predatory Pricing," Econometrica, 62 (1994), 1115-1140.

[7] Farrell, Joseph and Garth Saloner, "Installed Base: Compatibility, Innovation, Product Procurement, and Predation," American Economic Review, 76 (1986), 940-955.

[8] Fudenberg, Drew and David K. Levine, The Theory of Learning in Games, Cambridge: The MIT Press, 1998.

[9] Gale, Douglas and Robert W. Rosenthal, "Experimentation, Imitation, and Stochastic Stability," Journal of Economic Theory, 84 (1999), 1-40.

[10] Hehenkamp, Burkhard, C.-Z. Qin, and Charles Stuart, "Economic Natural Selection in Bertrand and Cournot Settings," Journal of Evolutionary Economics, 9 (1999), 211-224.

[11] Hehenkamp, Burkhard, "Sluggish Consumers: An Evolutionary Solution to the Bertrand Paradox," photocopy, 2000 (Games and Economic Behavior, forthcoming).

[12] Kandori, Michihiro, George Mailath, and Rafael Rob, "Learning, Mutation, and Long-Run Equilibria in Games," Econometrica, 61 (1993), 29-56.

[13] Katz, Michael L. and Carl Shapiro, "Network Externalities, Competition, and Compatibility," American Economic Review, 75 (1985), 424-440. 
[14] Pakes, Ariel, "A Framework for Applied Dynamic Analysis in I.O.," NBER Working Paper 8024, December 2000.

[15] Rhode, Paul and Mark Stegeman, "Non-Nash Equilibria of Darwinian Dynamics with Applications to Duopoly," International Journal of Industrial Organization, 19 (2001), 415-453.

[16] Rubinstein, Ariel, Modeling Bounded Rationality, Cambridge: MIT Press, 1998.

[17] Simon, Herbert A., Models of Bounded Rationality: Behavioral Economics and Business Organization, Volume 2, Cambridge: MIT Press (1982).

[18] Vega-Redondo, Fernando, "The Evolution of Walrasian Behavior," Econometrica, 65 (1997), 375-384.

[19] Vickers, John, "The Evolution of Market Structure When There is a Sequence of Innovations," Journal of Industrial Economics, 35 (1986), 1-13.

[20] Weisbuch, Gérard, Alan Kirman, and Dorothea Herreiner, "Market Organisation and Trading Relationships," Economic Journal, 110 (2000), 411-436.

[21] Young, H. Peyton, "The Evolution of Conventions," Econometrica, 61 (1993), 57-84. 
Figure 1

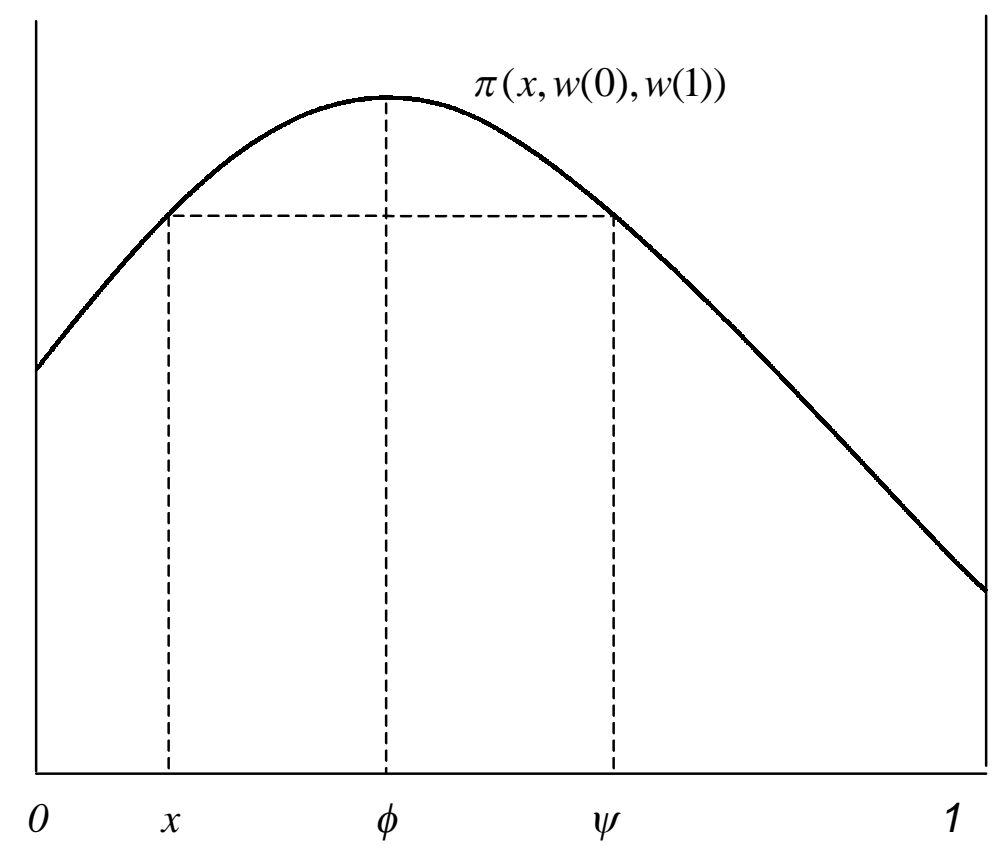

Figure 2

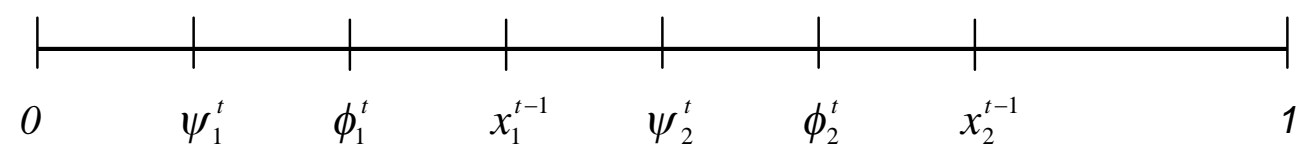


Figure 3: $\alpha=0.6, \omega=0.7$
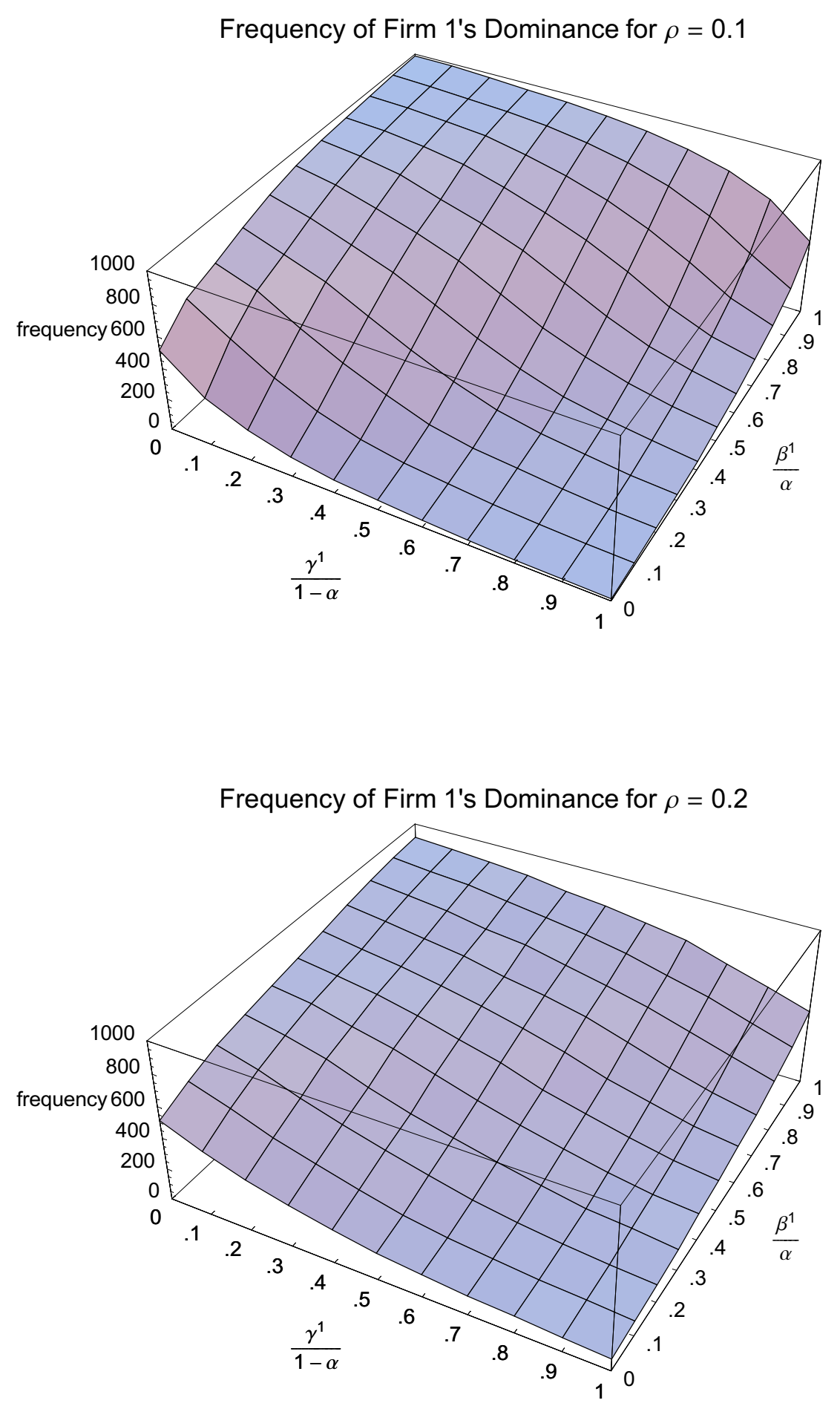


$$
\begin{gathered}
\text { Figure 4: } \alpha=0.6, \frac{\gamma^{1}}{1-\alpha}=0.5, \omega=0.7 \\
\left(x_{1}^{0}, x_{2}^{0}\right)=(x, 1-x)
\end{gathered}
$$

Frequency of Firm 1's Dominance for $\rho=0.3$

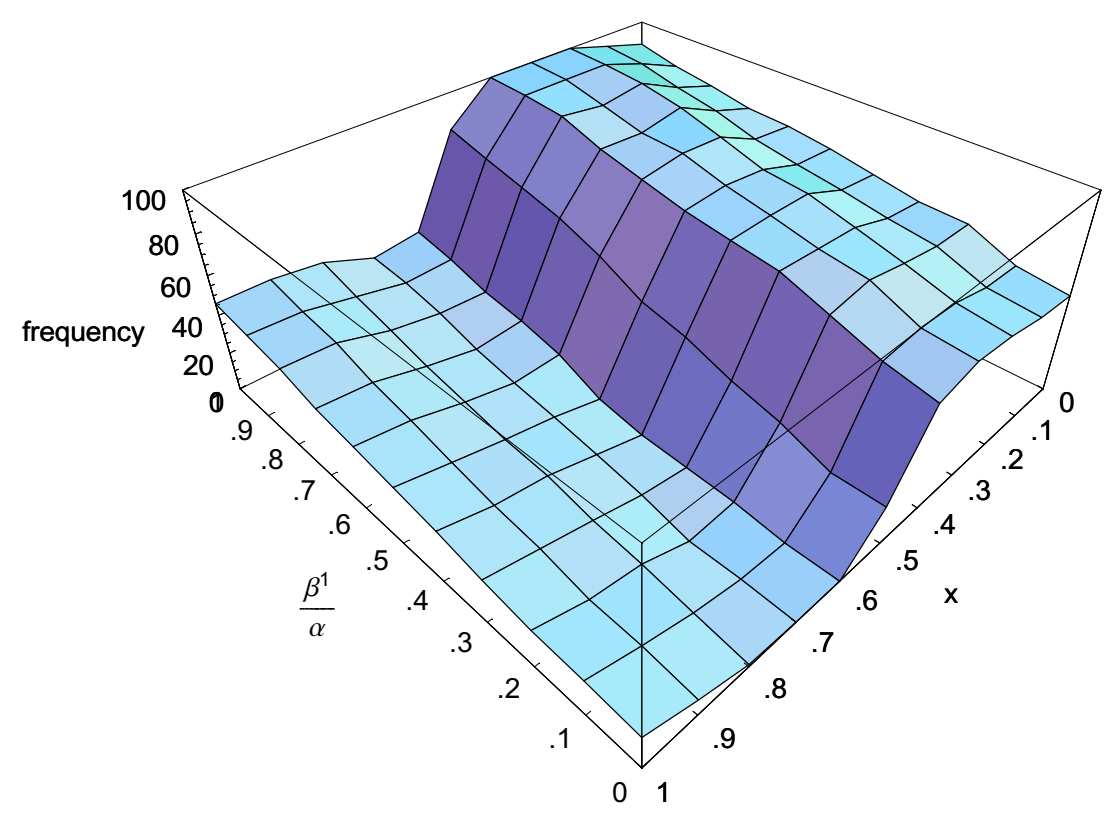

Frequency of Firm 1's Dominance for $\rho=0.4$

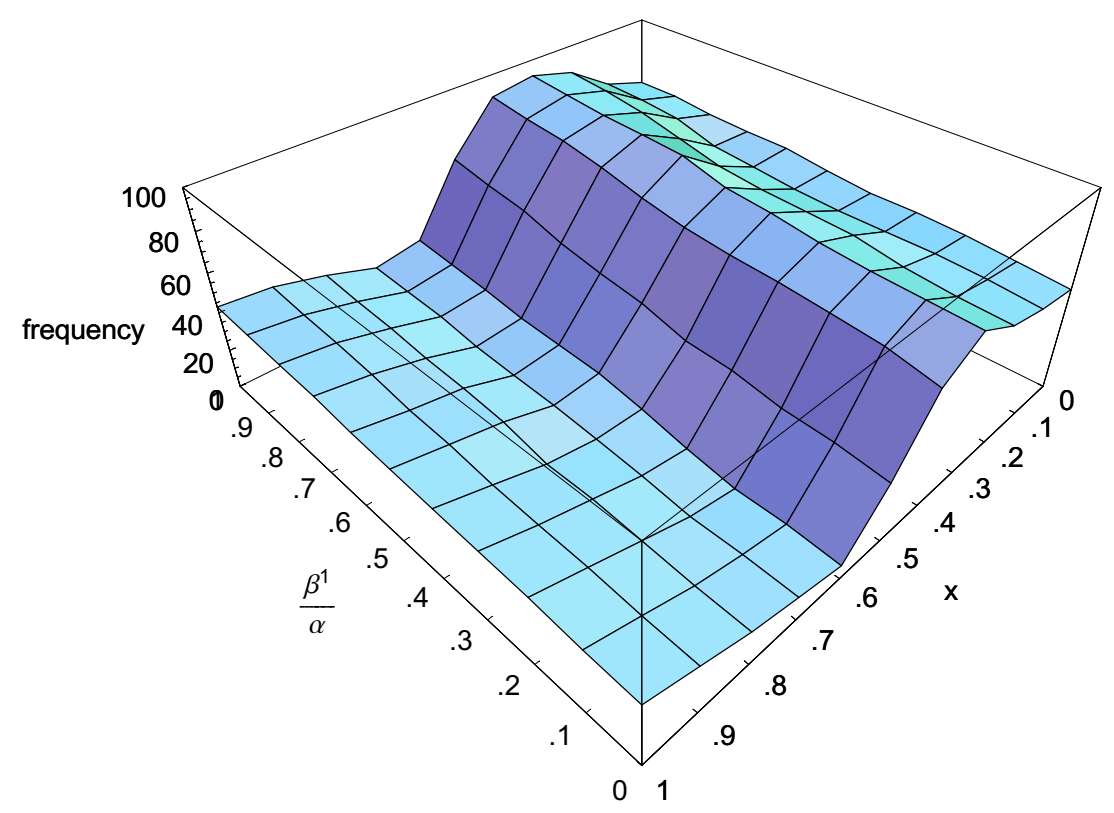


Figure 5: Impact of $\rho(\alpha=.6 ; \omega=.7)$

frequency of firm 1's dominance

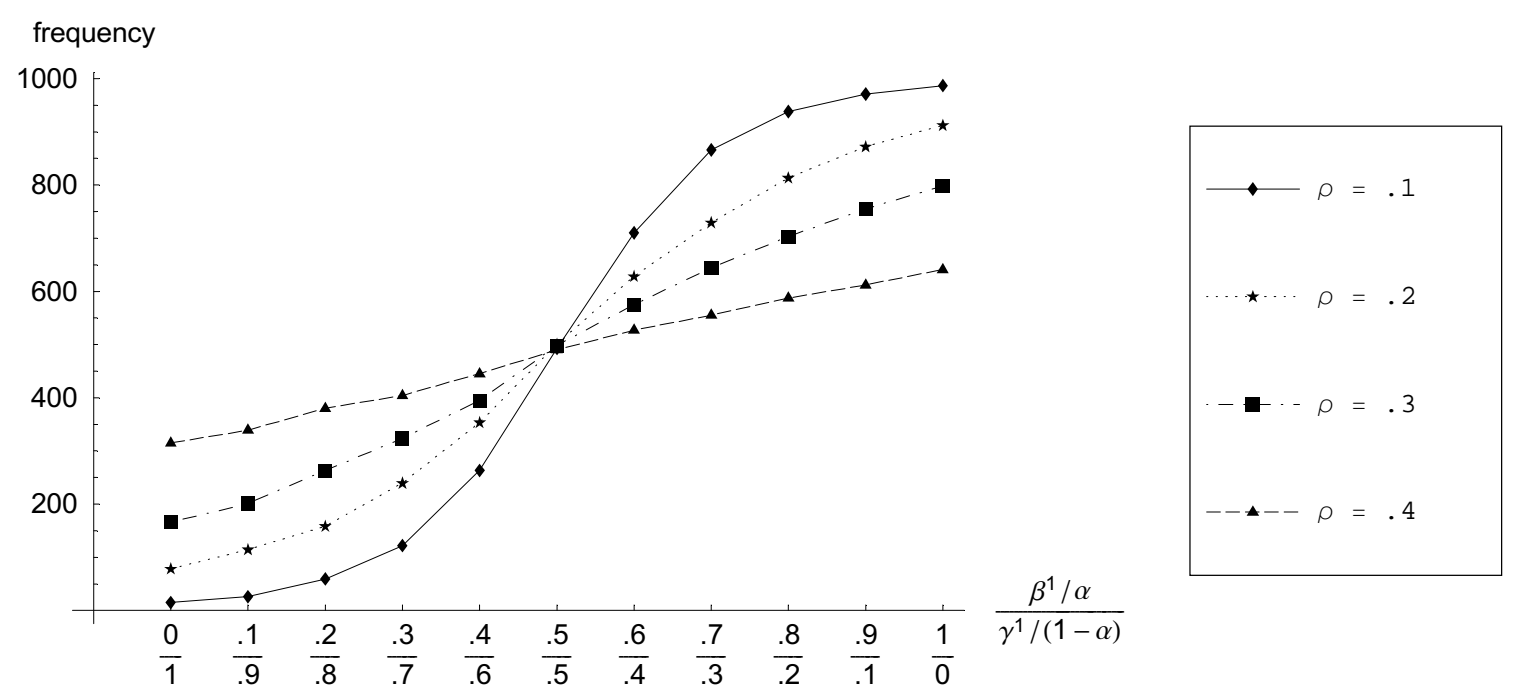

Figure 6: Impact of $\omega(\alpha=.6 ; \rho=.2)$

frequency of firm 1's dominance

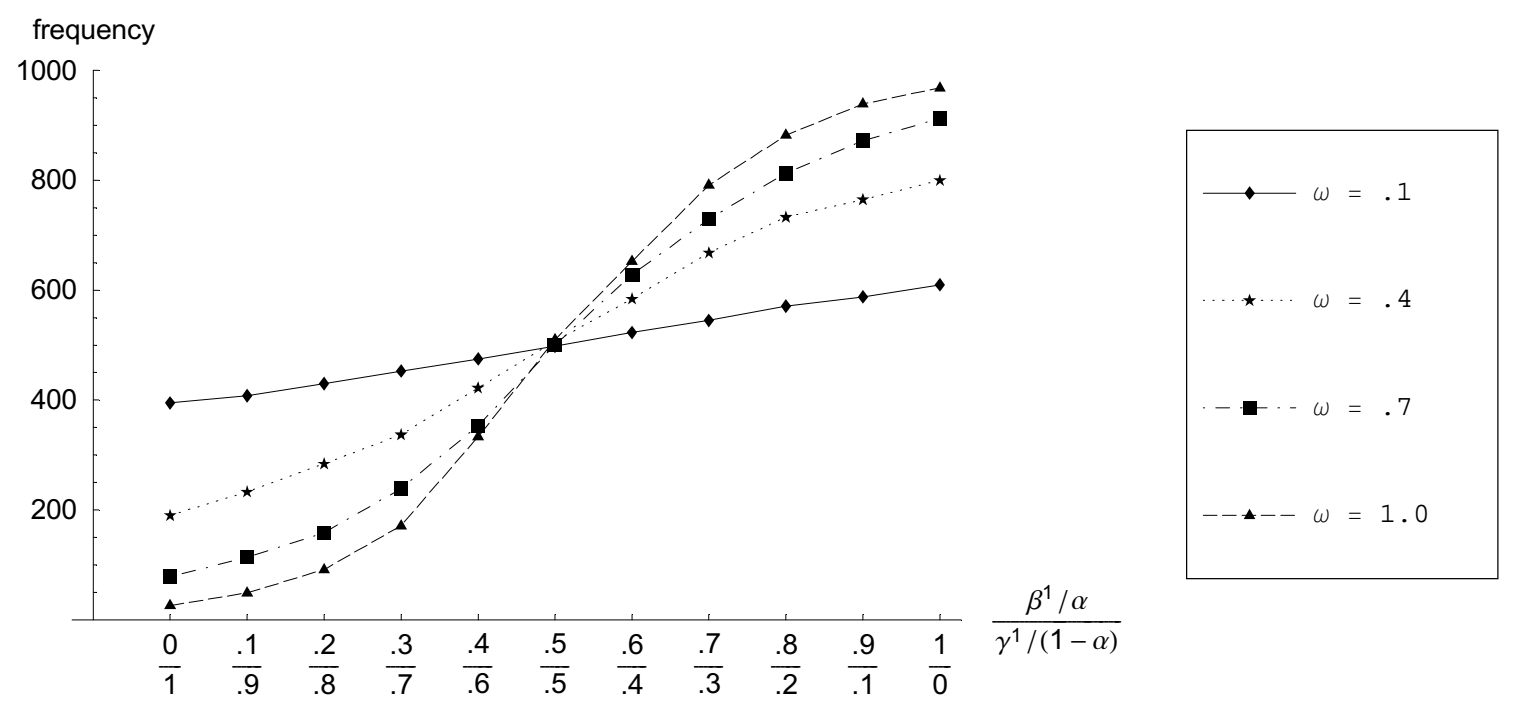

